\title{
Oyster (Crassostrea Virginica, Gmelin 1791) Population Dynamics On Public Reefs In The Great Wicomico River, Virginia, USA
}

Melissa Southworth

Virginia Institute of Marine Science

Juliana M. Harding

Virginia Institute of Marine Science

James A. Wesson

Roger L. Mann

Virginia Institute of Marine Science

Follow this and additional works at: https://scholarworks.wm.edu/vimsarticles

Part of the Marine Biology Commons

\section{Recommended Citation}

Southworth, Melissa; Harding, Juliana M.; Wesson, James A.; and Mann, Roger L., Oyster (Crassostrea Virginica, Gmelin 1791) Population Dynamics On Public Reefs In The Great Wicomico River, Virginia, USA (2010). Journal Of Shellfish Research, 29(2), 271-290.

$10.2983 / 035.029 .0202$

This Article is brought to you for free and open access by the Virginia Institute of Marine Science at W\&M ScholarWorks. It has been accepted for inclusion in VIMS Articles by an authorized administrator of W\&M ScholarWorks. For more information, please contact scholarworks@wm.edu. 


\title{
OYSTER (CRASSOSTREA VIRGINICA, GMELIN 1791) POPULATION DYNAMICS ON PUBLIC REEFS IN THE GREAT WICOMICO RIVER, VIRGINIA, USA
}

\author{
MELISSA SOUTHWORTH, ${ }^{1}$ JULIANA M. HARDING, ${ }^{1}$ JAMES A. WESSON ${ }^{2}$ \\ AND ROGER MANN ${ }^{1 *}$ \\ ${ }^{1}$ Department of Fisheries Science, Virginia Institute of Marine Science, College of William and Mary \\ PO Box 1346, Gloucester Point, VA 23062; ${ }^{2}$ Virginia Marine Resources Commission, \\ 2600 Washington Avenue, Newport News, VA 23607-0756
}

\begin{abstract}
We describe oyster population trends in the Great Wicomico River, VA, from 2000 through 2009 using quantitative fishery independent survey data collected using a stratified random design. The seven public reefs examined cover a total of $2.8 \times$ $10^{5} \mathrm{~m}^{2}$ and vary in individual size from $1.36 \times 10^{4}$ to $7.16 \times 10^{4} \mathrm{~m}^{2}$. The river is functionally divided by a sand spit into upriver and downriver regions. Oyster densities on the upriver reefs were typically an order of magnitude higher than densities on the downriver reefs within the same time period. Throughout the system, the highest observed densities were coincident with high annual recruitment events $(2002,2006)$. Recruitment events were usually followed by high mortality, with small percentages of the population reaching $\geq 3$ y of age. A predictive stock-recruit relationship is absent; rather, population demographics appear to be dominated by periodic high recruitment events. In the absence of seed removal, biomass maxima follow 1-2 y after recruitment maxima. Standing stock for the system varied between $1.56 \times 10^{6} \mathrm{~g}$ and $3.63 \times 10^{7} \mathrm{~g}$ in 2005 and 2006. Year-specific age-at-length relationships were estimated from demographics data. Length demographics were recast as age demographics to estimate mortality. Observed proportional mortality between young of the year and age 2 oysters was approximately 0.88 for the $2006-y$ class, which is slightly higher than the $0.62-0.71$ observed for the $2007-y$ class. The ability to estimate age specific mortality accurately allows the construction of shell (habitat) budgets for the individual reef systems. The Great Wicomico oyster population appears to be maintained by episodic and extraordinary recruitment in the face of high mortality - the latter driven by disease (predominantly Perkinsus marinus) epizootics. The shell resource is modest, equivalent to little more than a monolayer several centimeters thick. Over short timescales (years), the available shell resource oscillates in concert with mortality. The shell accretion rate on upriver reefs is consistently 4-5 times greater than that observed on downriver reefs. Periodic modest shell planting has maintained the habitat base (the shell resource) throughout the system over decadal scales.
\end{abstract}

KEY WORDS: Eastern oyster, Crassostrea virginica, population demographics, mortality, recruitment, shell budgets, Great Wicomico River

\section{INTRODUCTION}

The Great Wicomico River is a small Virginia estuary on the western shore of the Chesapeake Bay. It is characterized by a single, central deep channel; a sand spit protruding approximately halfway across its mouth; and a small watershed (approximately $337 \mathrm{~km}^{2}$, Chesapeake Bay Program; www.cheasapeake bay.net) containing predominantly forested and agricultural lands. The oyster habitat in the Great Wicomico River was originally mapped by Baylor $\left(1896^{1}\right)$ and was revisited by Haven et al. (1981). These areas, known as the Baylor Grounds or Public Oyster Grounds of Virginia, are held in public trust and are currently under the management of the Virginia Marine Resources Commission (VMRC).

Eastern oysters, Crassostrea virginica, created biogenic reef habitat in estuarine and coastal ecosystems that was sustained by sequential recruitment, growth, and mortality (Mann et al. 2009a). Over geological time, as the Chesapeake Bay was formed by a rising sea level, oyster larvae migrated into the developing estuaries from coastal waters and established the founding populations for massive reef systems that developed over thousands of years (Hargis 1999, Smith et al. 2003). The positive relationship between live oysters and live shell habitat is well documented (Powell et al. 2006, Powell \& Klinck 2007, Mann et al. 2009a, Mann et al. 2009b). Fishing removes both habitat

*Corresponding author. E-mail: rmann@vims.edu and live oysters. More than three centuries of fishing pressure have drastically reduced the spatial footprint and vertical relief of Chesapeake oyster populations relative to what they were before European settlement (e.g., Haven et al. 1981, Smith et al. 2003, Woods et al. 2005) in the Great Wicomico River and the bay as a whole.

Andrews (1979) described the Great Wicomico River as a trap-type estuary with a gyrelike circulation that historically contributed to high recruitment of oysters within the system and its development as a seed river since the 1960s (Haven et al. 1978). Southworth and Mann (2004) described patterns of recruitment in the river from the early 1960s through the early 2000s. Such trap-type estuaries were historically used by VMRC and the Virginia oyster industry for the planting of shell prior to annual oyster recruitment. The subsequent harvest and relocation of that shell with attached recruits (commonly termed "seed oysters") to other locations (Haven et al. 1978, Haven et al. 1981) in the Virginia portion of the Chesapeake Bay for grow-out to market size and eventual harvest has been and continues to be included in management and replenishment efforts for surviving native oyster populations.

The Great Wicomico River has a history of repletion (for fisheries) and restoration (for ecological services) activity. VMRC has managed this estuary as a source of seed oysters since 1963 (Haven et al. 1978). Repletion efforts include both seed harvest (early spring) and shell planting (early summer) on public reefs (Haven et al. 1978, Haven \& Whitcomb 1986). Prior to 1963 and the onset of shell planting by VMRC, natural cultch 
sufficient to catch a set relevant to commercial production was considered lacking (Haven et al. 1978) . In 1996, a 3-dimensional reef (Shell Bar Reef, CR1, Fig. 1) was built in the system, and later received broodstock oysters to enhance larval production and recruitment in the system (Southworth \& Mann 1998). Threedimensional reefs have enhanced vertical relief (up to several meters) compared with the typical "shell plant" or 2-dimensional reef common to fishery maintenance practices. Shell plants with uniform depths of shell overlay are built by VMRC, typically with shell cover rates of 1,000 bushels per acre every $5 \mathrm{y}$, equivalent to a uniform addition of $12 \mathrm{~L} / \mathrm{m}^{2}$. A second 3-dimensional reef (Crane's Creek, CR2, Fig. 1) was built in 1997 (Southworth et al. 2000). As a method of oyster reef enhancement and management, Hargis and Haven (1999) recommended the building of 3-dimensional sanctuary reefs surrounded by 2dimensionsal shell plants for market and/or seed oyster production. Following the building of the 3-dimensional reefs in the system, several of the adjacent Public Oyster Grounds have received additions of shell substrate (Dr. James Wesson, VMRC Conservation and Replenishment Division annual unpublished replenishment reports). These "shell plants" have been used by VMRC to support Virginia's public oyster fishery in both the movement of seed from the Great Wicomico to public grounds in other systems and, to a limited extent, the production of market oysters within the river.

In addition to the Baylor Grounds in the Great Wicomico River, there are also areas of bottom that private citizens lease from the state (Haven et al. 1981). Activities on the private leased areas include shell plants to enhance recruitment as well as seed movement from the system in a similar fashion to that used on the public grounds. Since at least 2007, aquaculture businesses have placed oysters in cages for grow-out on their leased bottoms in the Great Wicomico (A.J. Erskine, Cowart Seafood Corporation, pers. comm.). The total area of productive

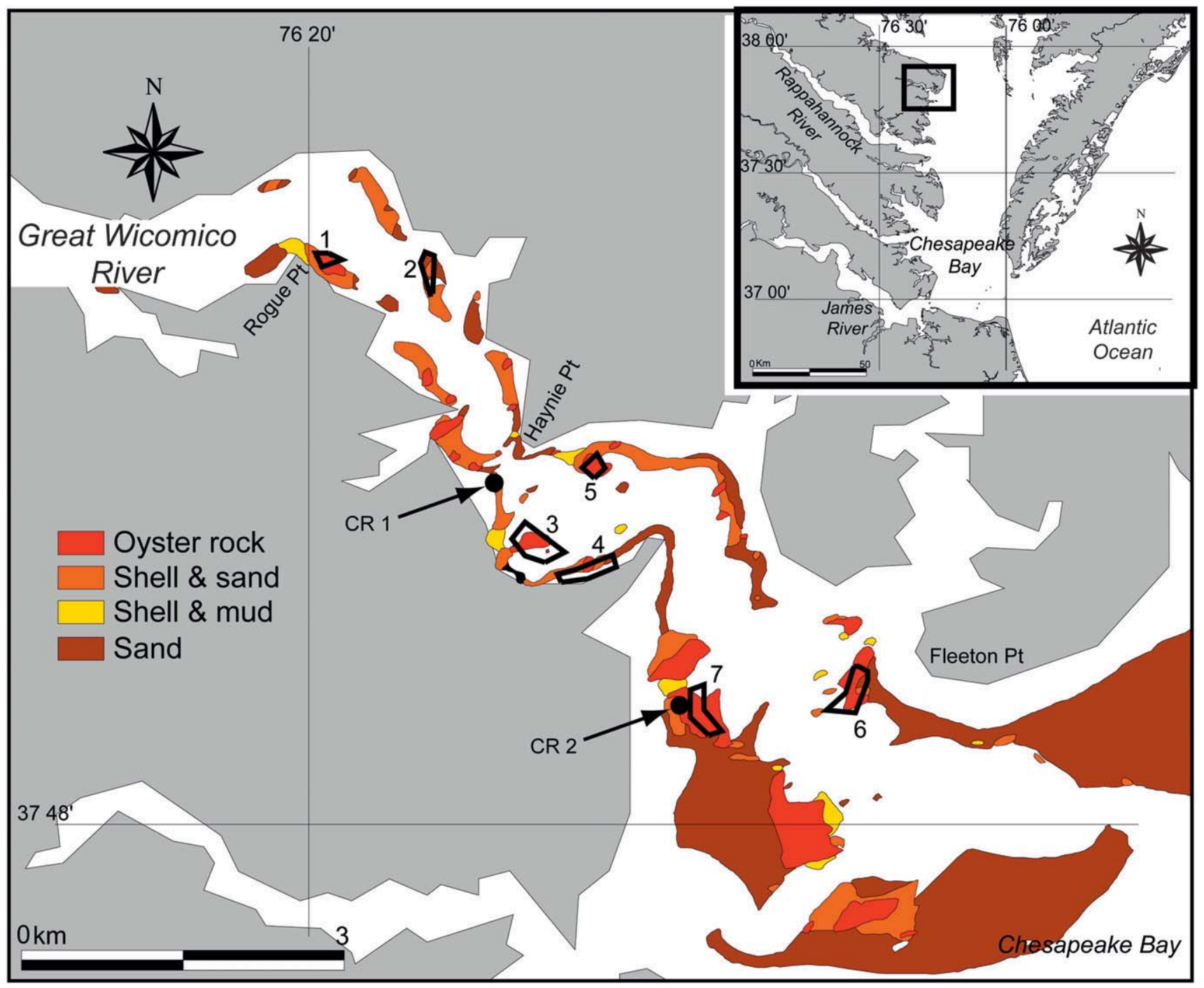

Figure 1. Map showing the locations of seven public oyster reefs in the Great Wicomico River, VA, sampled with patent tongs annually from 2000 to 2009. (1) Rogue Point, (2) Harcum Flats, (3) Shell Bar, (4) Sandy Point, (5) Haynie Point, (6) Fleet Point, and (7) Cranes Creek. The underlying habitat categorizations (oyster rock, shell and sand, shell and mud, sand) are from Haven et al. (1981). The CR notation identifies 3-dimensional constructed oyster reefs CR1 (Shell Bar Reef, 1996) and CR2 (Cranes Creek Reef, 1997), as discussed in the text. The public reef numbering system will be used throughout the text and figures. 
oyster bottom in the Great Wicomico River covered by private leases is currently estimated to be 2-3 times greater than the productive area on the public grounds, but reporting is not mandatory and statistics are not available.

The goal of Virginia's public oyster resource management effort is to sustain both oyster productivity and the integrity of the habitat. Implementation of actions in support of this goal is, however, challenging in that the included elements of rebuilding broodstock, sustaining the habitat (shell) base, and harvesting seed for relocation can be in conflict. For example, harvest of seed entails removal of habitat, thus requiring eventual supplementation of the latter by additional shell planting. In this contribution we examine sequential changes in oyster population demographics and habitat stability on seven Great Wicomico River public oyster reefs from 2000 through 2009.

\section{MATERIALS AND METHODS}

Survey methods for the current Great Wicomico River study follow those described in Mann et al. (2009b) for oysters in the James River, VA. In the current study, oysters were collected during the fall (November) from seven natural oyster reefs within public grounds from 2000 through 2009 in the Great Wicomico River (Fig. 1). A quantitative sampling program used a stratified random grid, with individual oyster reefs (bars) forming the strata (Mann et al. 2009b). Reef names and locations for the public reefs (Fig. 1) adhere to those used by Baylor (1896) and subsequently resurveyed by Haven et al. (1981).

\section{Temperature and Salinity}

Water temperature was collected on a weekly basis from June through September at six sites (reefs 1, 2, 3, 5, 6, and 7 in Fig. 1) throughout the study. From 2000 to 2004, water samples were collected approximately $0.5 \mathrm{~m}$ off the bottom, and temperature was measured with an alcohol thermometer within 5 min of water sample collection. Beginning in 2005, water temperature was measured with a hand-held digital probe (YSI 85, Yellow Springs Instruments, Yellow Springs, CO) suspended $0.5 \mathrm{~m}$ from the bottom. Data collected within a single week were consistently within a $1^{\circ} \mathrm{C}$ range and were averaged across all sites. Beginning in June 2005, temperature was also measured at 15-min intervals with a YSI 600 (Yellow Springs Instruments, Yellow Springs, CO) series sonde mounted $0.5 \mathrm{~m}$ above the bottom at CR1 (Fig. 1). A linear regression between Great Wicomico (Great Wicomico temperature $=\mathrm{b}+\mathrm{m}$ (York temperature)) measured water temperatures at CR1 and a similar sonde deployed in the York River (Gloucester Point, VA, $37^{\circ} 14^{\prime} 47^{\prime \prime} \mathrm{N}, 76^{\circ} 30^{\prime} 23^{\prime \prime} \mathrm{W}$ ) was used to predict water temperatures at CR1 prior to June 2005, where data are lacking from the Great Wicomico River but available for the York River.

Salinity data were collected commensurate with weekly water temperature data from June through September 2000 to 2009. From 2000 to 2004, salinity (measured in ppt) in water samples collected with a Niskin bottle $0.5 \mathrm{~m}$ from the bottom was measured with a hand-held refractometer. From 2004 to 2009, weekly salinities were measured with a hand-held digital conductivity probe (YSI 85) located approximately $0.5 \mathrm{~m}$ above the bottom.

Linear regressions $(\mathrm{GW}$ salinity $=\mathrm{b}+\mathrm{m}$ (York salinity)), where GW is Great Wicomico, were used to relate average daily salinity data from the York River at Gloucester Point and CR1 in the Great Wicomico River for each month (12 total regressions) from June 2005 through 2009. These regressions were then used with measured average daily salinities from the York River at Gloucester Point from Jan 2000 through May 2005 to predict the salinities in the Great Wicomico River.

\section{Oyster Field Collections}

Oysters were collected from the 43-ft-long VMRC vessel J.B. Baylor with a hydraulic patent tong. The open dimensions of the tong were such that it sampled $1 \mathrm{~m}^{2}$ of bottom. Upon retrieval of each sample (= patent tong grab), oysters were counted and measured (in millimeters), and the volume of shell material (measured in liters) recorded. The recorded dimension was the longest from the hinge to the shell margin. This is correctly termed "shell height," although commonly described as shell length (SL) in most literature. We adopt the common convention and refer to SL in subsequent text. A count of the number of oysters per tong was made in all years sampled. From 2000 through 2002, all oysters were measured and classified into 5 -mm-size bins. Beginning in 2003, for each sample, individual oyster lengths were recorded to the nearest millimeter. Samples with more than $20 \mathrm{~L}$ of shell were halved to facilitate processing. The resulting counts and length frequency distributions for the subsample were doubled to estimate density and size distribution on a per square meter basis when subsampling was necessary. The procedures of Bros and Cowell (1987) were used to ensure adequacy of sampling within each strata.

Average oyster density (measured in number per square meter) was calculated for each oyster reef by averaging the number of oysters collected from all samples on a reef within a year. Average shell volume (measured in liters per square meter) was calculated by averaging the total volume of shell collected from all samples on a reef within a year. For 2002 through 2009, shell was additionally categorized as brown shell (shell that lies above the sediment water interface) and black shell (shell that was exhumed during the collection process).

\section{Biomass Estimation}

Data from a size range (16.5-98.5 $\mathrm{mm} \mathrm{SL})$ of live oysters $(n=$ 172) collected from the Great Wicomico River in November 2005 and 2006 were used to estimate the relationship between oyster SL (measured in millimeters) and biomass or dry tissue weight (measured in grams) on public oyster reefs. After oyster SL was measured to the nearest millimeter, the tissue was removed and dried to constant weight (dry weight (DW), measured in grams) at $80^{\circ} \mathrm{C}(72 \mathrm{~h})$.

Wet shell weight (WSW, measured in grams) was obtained from 398 oysters (16.5-115.6 mm SL), collected from 2004 through 2007, after the tissue had been removed and before the shells had dried. The relationship between SL and WSW was described. Biomass and shell weight calculations were made for each reef using the midpoint of each reef-specific, 5-mm size class as SL in the fitted SL-DW equation and are reported for 2000 through 2009.

\section{Age Structure and Mortality}

The estimation of age structure from length demographic plots follows the procedure in Harding et al. (2008) and Mann et al. (2009b). Briefly, demographic plots were prepared each 
year (2003 to 2009) for the live oysters ( $n=22,253$ oysters) measured from each reef, where live oysters were aggregated on a year-specific length frequency graph using 2-mm length bins. Individual cohorts were identified by the method of Bhattacharya (1967). The range and modal length of each cohort was identified by counting cohorts and relating the cohort settlement dates to long-term recruitment patterns developed from annual recruitment (spatfall) reports for the Great Wicomico River over the study period (Southworth et al. 2001, Southworth et al. 2002, Southworth et al. 2003, Southworth et al. 2004, Southworth et al. 2005, Southworth et al. 2006, Southworth et al. 2007, Southworth et al. 2008, Southworth et al. 2009, Southworth et al. 2010; available at http://www.vims.edu/mollusc). Mann et al. (2009b) assigned cohorts to years using a linear age-at-length relationship fit to the data ( $\mathrm{SL}=\mathrm{b}+\mathrm{m} \times$ Age) in preference to a von Bertalanffy (1938) model. The current data were examined using both a linear and a quadratic $\left(\mathrm{SL}=a \times(\text { Age })^{2}+b \times(\right.$ Age $\left.)+c\right)$ model for public reef collections. The quadratic relationship was chosen because it provides slightly better $R^{2}$ values and fit for the older age classes. Using the year-specific quadratic relationships, the demographic plots were recast as graphs of year classes for each year by location in the river for 2000 through 2009 . Recasting of the data from 2000 through 2002 (5-mm bins) for public reefs used the age-at-length relationship for 2003 .

Mortality, as a proportion of the standing population, was estimated by the following relationship:

$$
\text { Mortality }=[\# \operatorname{Live}(t)-\# \operatorname{Live}(t+1) / \# \operatorname{Live}(t)]
$$

where \#Live $(t)$ equals the number of live oysters at time $t(t$, units of $1 \mathrm{y}$ ). Possible errors in this approach are discussed in Mann et al. (2009b). Mortality on public reefs was investigated by aggregating data for reefs 1 through 5 (Fig. 1, hereafter termed "upriver reefs"), reefs 6 and 7 (hereafter termed "downriver reefs"), and all reefs within the river combined.

\section{Disease Status}

The prevalence and intensity of Perkinsus marinus (Dermo) and Haplosporidium nelsoni (MSX) at selected locations in the study area are reported by Ragone Calvo and Burreson (2001, 2002, 2003), Carnegie et al. (2004), and Carnegie and Burreson (2005, 2006, 2009). Disease data are presented herein as either prevalence (percent infected) or weighted prevalence (WP) for comparison with mortality data. Weighted prevalence is calculated based on the following formula from Burreson et al. (1988):

$$
W P=(0.5 \times R)+(1 \times L)+(3 \times M)+(5 \times H) / n
$$

where $R, L, M$, and $H$ are rare, light, medium, and heavy infection intensity, respectively; and $n$ is the total number of oysters tested. Disease samples are typically taken from oysters with an SL larger than $60 \mathrm{~mm}$.

\section{Data Analyses}

Significance levels for all tests were established at alpha $=$ 0.05 a priori. When appropriate, Fisher's parametric post hoc multiple comparison test was used.

A total of 490 data pairs (total volume, density) including patent tong grabs with total volume $=0$ and/or density $=0$ were used to describe the relationship between the presence of oyster shell and live oysters on public reefs in the Great Wicomico River (2000 through 2009) with a linear model:

$$
\text { Density }=0.4629+5.4701 \times(\text { Total shell volume })
$$

where total shell volume is measured in liters per square meter, $n=490$, and $R^{2}=0.17$.

The low y-intercept describes a prerequisite for oyster shell (habitat) prior to live oysters. All subsequent analyses include only tongs with shell volume greater than 0 and density of live oysters greater than $0(n=419$ tongs with nonzero total volume and density).

The data set for total shell volume (measured in liters per square meter) by reef (reef 7) and year (2000 through 2009) was too unbalanced to be analyzed with a 3-way analysis of variance (ANOVA; reef, river location, year); thus, a 2-way ANOVA (river location, year) was used to describe the effects of reef location (upriver or downriver) with regard to Sandy Point (Fig. 1), a sand spit that extends halfway across the mouth of the river, and year on total shell volume (measured in liters) between 2000 and 2009. Total shell volume data met assumptions of homogeneity of variance and normality without transformation.

Density and biomass data satisfied assumptions of normality and homogeneity of variance after transformation (natural logarithm). An analysis of covariance (ANCOVA) with total shell volume (measured in liters per square meter for 2000 through 2009) as a covariate, and location and year as factors, was used to evaluate density and biomass data (one ANCOVA per data set).

The relationship between total shell volume and brown shell volume was examined with 319 data pairs, where total shell and brown shell volume (2002 through 2009) were greater than 0. A linear model was used to describe the relationship between total shell volume and brown shell volume, where

Brown shell volume $=-1.22+0.709 \times($ Total shell volume $)(4)$

where brown shell volume and total shell volume are measured in liters per square meter and $R^{2}=0.64$. The negative intercept is indicative of a minimum basal or spatial footprint requirement of total shell volume to provide some brown shell (habitat) at the substrate-water interface resulting from the relatively fast rate of shell sinking below the redox level given the absence of natural hard substrate (e.g., historic terraces and scarps (Hobbs 2004)) in the Great Wicomico system. A minimum of approximately $2 \mathrm{~L}$ total shell volume is required to provide any positive value of brown shell volume.

Density and biomass data from sites with brown volume greater than 0 were analyzed with separate ANCOVAs, with brown shell volume (measured in liters per square meter for 2002 through 2009) as a covariate, and location and year as factors. Density data were log-transformed prior to analyses to satisfy the assumptions of normality and homogeneity of variance. Biomass data also satisfied these assumptions after transformation $(\operatorname{logarithm}+1)$.

\section{RESULTS AND DISCUSSION}

\section{Temperature and Salinity}

Annual variation in average weekly bottom water temperature for the Great Wicomico River is illustrated in Figure 2. There is good agreement between the Great Wicomico predictions (Table 1, 2000 through 2004), weekly data (May through October 2000 through 2009), and sonde (2005 through 2009) data series for the Great Wicomico. Great Wicomico River 


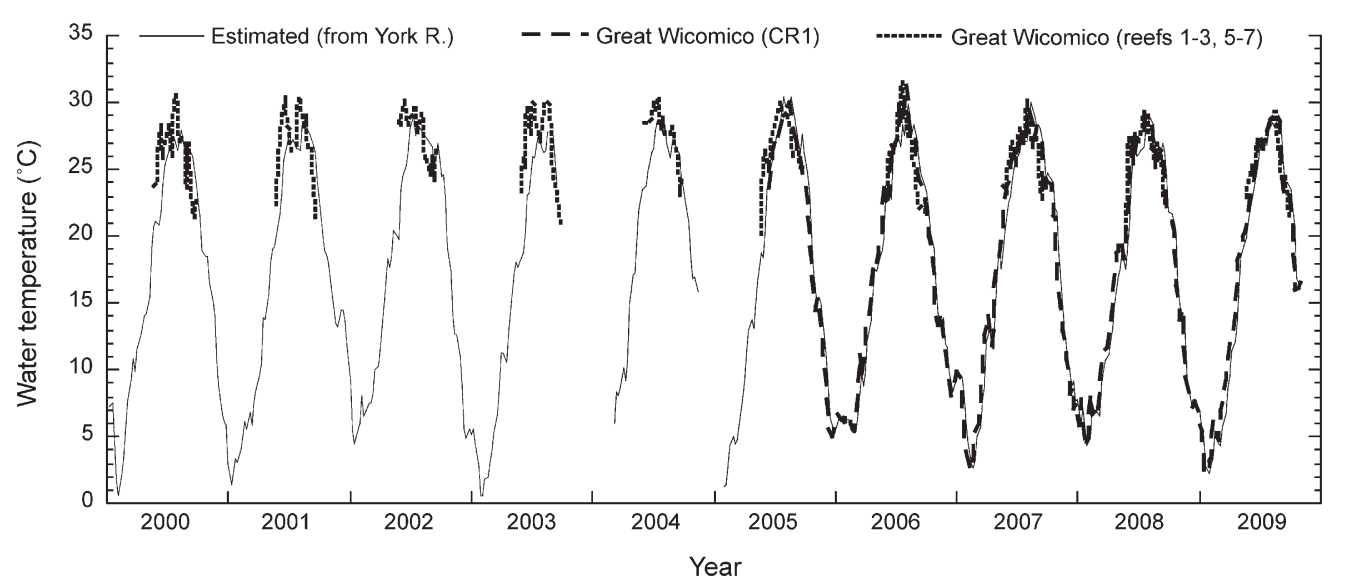

Figure 2. Average weekly bottom temperature in the Great Wicomico River, VA, as estimated from the York River (VIMS, Gloucester Point, solid line), measured continuously at CR1 (2005 through 2009; dashed line), and weekly measurements averaged across reefs 1-3 and 5-7 (June through September 2000 through 2009; dotted line).

summer maxima consistently reach or exceed $30^{\circ} \mathrm{C}$ in all years except 2008 and 2009 (Fig. 2). Winter minima in the Great Wicomico River reached a low of $2^{\circ} \mathrm{C}$ in the winters of $2006 / 2007$ and $2007 / 2008$. Winter minima in the Great Wicomico River in 2005/2006 and 2007/2008 remained at or above $5^{\circ} \mathrm{C}$. (Fig. 2).

Salinity records for the Great Wicomico River for the study period are illustrated in Figure 3. As with temperature, estimates based on York River data are used when Great Wicomico field data are unavailable (Table 1). Early summer salinity in 2000 was 10 ppt and thereafter rose gradually to late fall values of approximately 15 ppt. Summer 2001 observations were of higher salinity, in the 13-19-ppt range, whereas summer 2002 values were yet higher, in the 17-22-ppt range. The summers of 2003 and 2004 were less saline at $10-15$ ppt. Late-summer salinities in 2005 through 2009 were consistently in excess of 13 ppt, rising throughout the late summer to fall to 16-19 ppt. Winter salinities in the Great Wicomico were at or above 13 ppt in 2005 to 2006, approximately 10 ppt in 2006 to 2007 and 2007 to 2008, and at or above $14 \mathrm{ppt}$ in the winter to spring of 2008 to 2009. Salinity minima are typically seen in the March through May period in the Great Wicomico River.

\section{Description of the Oyster and Shell Resource}

\section{Oyster Density and Biomass}

Changes in reef-specific oyster density for 2000 through 2009 are summarized in Table 2. The area of the individual reefs (sampling strata) varied by a factor of $4\left(1.36 \times 10^{4}\right.$ to $5.90 \times$ $10^{4} \mathrm{~m}^{2}$ ). Reefs 1 through 5 border the river channel upriver of Sandy Point (Fig. 1). Reefs 6 and 7 lie downstream of the sand spit in close proximity to the Chesapeake Bay.

Oyster densities (measured per square meter) and biomass (measured in grams of dry tissue per square meter; Table 3) upriver of Sandy Point were significantly higher than densities and biomass downstream of the sand spit (ANCOVAs, Table

TABLE 1.

Linear regression coefficients (and error terms) used to predict Great Wicomico River water temperatures and salinities from January 1, 2000, through October 31, 2009, from data measured in the York River and Great Wicomico River (at CR1) between June 14, 2005 and December 31, 2008, when monitoring stations were established in both systems.

\begin{tabular}{|c|c|c|c|c|c|c|c|}
\hline Relationship & Month & $n$ & $\mathbf{m}$ & SE $\mathrm{m}$ & b & SE b & $\overline{R^{2}}$ \\
\hline Water temperature & NA & 1,222 & 1.0684 & 0.0028 & -1.3544 & 0.0531 & 0.99 \\
\hline \multirow[t]{12}{*}{ Salinity } & Jan & 92 & 0.7713 & 0.0362 & -0.0650 & 0.7194 & 0.83 \\
\hline & Feb & 76 & 1.0022 & 0.0790 & -5.9712 & 1.5690 & 0.68 \\
\hline & Mar & 93 & 0.9260 & 0.0989 & -4.3676 & 1.8370 & 0.49 \\
\hline & Apr & 90 & 0.7528 & 0.0272 & -0.7590 & 0.4757 & 0.90 \\
\hline & May & 91 & 0.9282 & 0.0565 & -3.6571 & 0.9715 & 0.75 \\
\hline & June & 88 & 0.8550 & 0.0731 & -2.6556 & 1.3775 & 0.61 \\
\hline & July & 102 & 0.3290 & 0.0964 & 7.6857 & 1.9774 & 0.10 \\
\hline & Aug & 118 & 0.6646 & 0.0584 & 1.0589 & 1.2248 & 0.53 \\
\hline & Sept & 104 & 0.5106 & 0.0495 & 5.8655 & 1.0478 & 0.51 \\
\hline & Oct & 124 & 0.6322 & 0.0357 & 3.9218 & 0.7656 & 0.72 \\
\hline & Nov & 120 & 0.8740 & 0.0218 & -1.1968 & 0.4686 & 0.93 \\
\hline & Dec & 122 & 1.1029 & 0.0442 & -6.4896 & 0.9259 & 0.84 \\
\hline
\end{tabular}

$n=$ the number of $x$ and $y$ data pairs used in the regression; $\mathrm{m}=$ slope of the line; SE $\mathrm{m}=$ the $\mathrm{SE}$ for $\mathrm{m} ; \mathrm{b}=$ the $\mathrm{y}$-intercept; $\mathrm{SE} \mathrm{b}=$ the $\mathrm{SE}$ error for $\mathrm{b}$; $R^{2}=$ the coefficient of determination; NA = not applicable. 


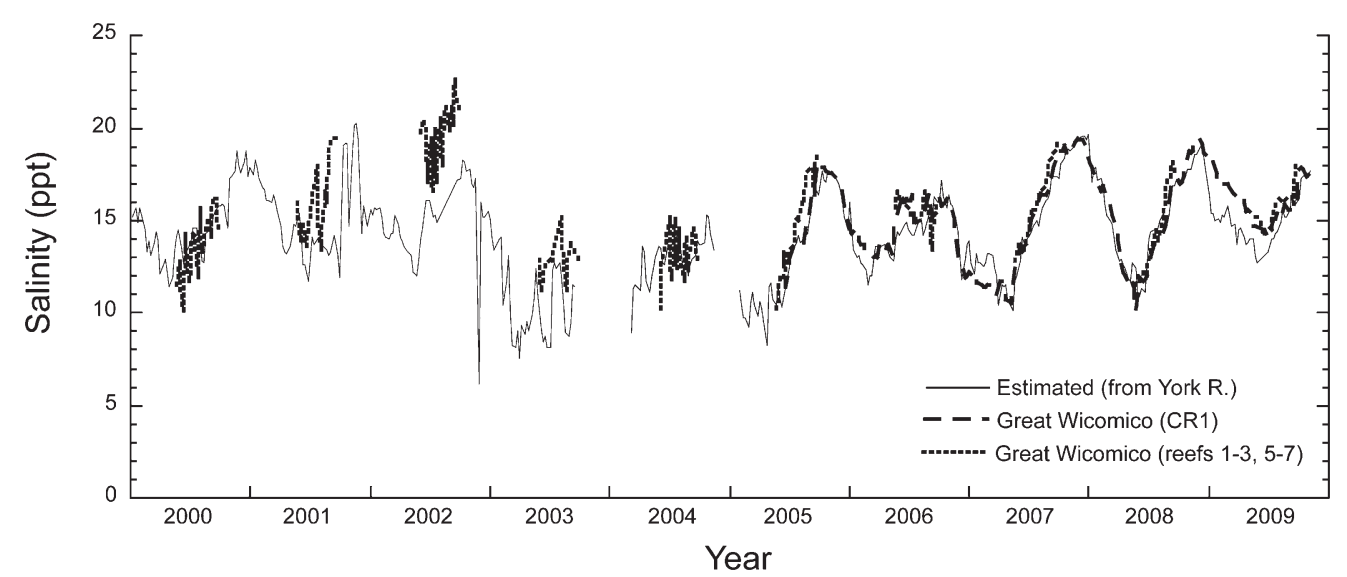

Figure 3. Average weekly bottom salinity in the Great Wicomico River, VA, as estimated from the York River (VIMS, Gloucester Point, 2000 through 2009; solid line), measured continuously at CR1 (2005 through 2009; dashed line), and weekly measurements averaged across reefs 1-3 and 5-7 (June through September 2000 through 2009; dotted line).

4). In general, oyster density and biomass data sets followed similar trends with regard to location within the river and year. Both oyster density and biomass were significantly affected by year, location within the river, and total shell volume (Table 4, ANCOVA with total shell volume as a covariate) as well as brown shell volume (Table 4, ANCOVA with brown shell as a covariate). Biomass was significantly higher in 2007 through 2009 than in all other years, reflecting the growth of the 2006-y class. Densities in 2006 were higher than all other years examined.

Reefs upriver of Sandy Point had significantly more total shell volume (Table 5) than sites downriver (ANOVA, Table 4). Total shell volume was significantly higher in 2009 than in 2004, 2005, and 2006. These differences are related to the removal of seed in spring 2003 (which has a cascading effect on demographics and shell dynamics for several years past the event, as described later) combined with the maturation of the 2006-y class by 2009 .
Reefs upriver of Sandy Point (Table 2, Fig. 1, reefs 1-5) can be generally described as a group based on density of live oysters. Mean densities are generally in the range of $10-50 / \mathrm{m}^{2}$ in 2000, 2001, and 2003 through 2005. Higher densities were observed in $2002\left(62.3-284.4 / \mathrm{m}^{2}\right)$ and in 2006 through 2009 (54.3-595.6/ $\mathrm{m}^{2}$, Table 2). A trend of generally decreasing density from 2003 through 2005 followed the 2002 recruitment event. 2006 was the highest density in the 2006 through 2009 period at reefs 1,3 , and 5. At the downstream reefs, the highest densities at reef 6 were recorded in 2002 and 2007, with the highest densities at reef 7 recorded in 2006 through 2009 (Table 2).

Spatial trends in oyster distribution within reefs can be examined through variance-to-mean ratios (Fig. 4, ratios greater than 1 indicate aggregation, ratios equal to 1 describe a random distribution, values less than 1 indicate a uniform distribution). Most (87\%) of the observed variance to mean

TABLE 2.

Average oyster density on the seven Great Wicomico River public reefs from 2000 through 2009.

\begin{tabular}{|c|c|c|c|c|c|c|c|c|c|c|c|}
\hline $\begin{array}{c}\text { Station, } \\
\text { Reef Name }\end{array}$ & Area $\left(m^{2}\right)$ & 2000 & 2001 & 2002 & 2003 & 2004 & 2005 & 2006 & 2007 & 2008 & 2009 \\
\hline 1) Rogue Point & $1.36 \times 10^{4}$ & $\begin{array}{c}49.9 \\
(19.2)\end{array}$ & $\begin{array}{l}16.9 \\
(9.9)\end{array}$ & $\begin{array}{c}284.4 \\
(67.3)\end{array}$ & $\begin{array}{c}34.9 \\
(11.1)\end{array}$ & $\begin{array}{l}16.4 \\
(6.2)\end{array}$ & $\begin{array}{l}11.1 \\
(3.9)\end{array}$ & $\begin{array}{c}595.6 \\
(114.0)\end{array}$ & $\begin{array}{c}298.0 \\
(45.9)\end{array}$ & $\begin{array}{c}248.1 \\
(44.4)\end{array}$ & $\begin{array}{c}94.1 \\
(35.0)\end{array}$ \\
\hline 2) Harcum Flats & $2.42 \times 10^{4}$ & $\begin{array}{c}37.9 \\
(15.0)\end{array}$ & $\begin{array}{l}38.4 \\
(7.9)\end{array}$ & $\begin{array}{l}198.1 \\
(35.1)\end{array}$ & $\begin{array}{c}33.6 \\
(11.5)\end{array}$ & $\begin{array}{l}16.0 \\
(6.5)\end{array}$ & $\begin{array}{c}5.1 \\
(2.0)\end{array}$ & $\begin{array}{c}163.4 \\
(39.9)\end{array}$ & $\begin{array}{l}244.0 \\
(34.2)\end{array}$ & $\begin{array}{l}148.3 \\
(25.0)\end{array}$ & $\begin{array}{l}120.3 \\
(13.9)\end{array}$ \\
\hline 3) Shell Bar & $7.16 \times 10^{4}$ & $\begin{array}{c}38.1 \\
(11.5)\end{array}$ & $\begin{array}{l}16.9 \\
(5.8)\end{array}$ & $\begin{array}{c}62.3 \\
(19.1)\end{array}$ & $\begin{array}{c}12.9 \\
(5.0)\end{array}$ & $\begin{array}{l}18.4 \\
(6.1)\end{array}$ & $\begin{array}{c}7.1 \\
(3.2)\end{array}$ & $\begin{array}{c}183.4 \\
(37.8)\end{array}$ & $\begin{array}{l}109.4 \\
(42.5)\end{array}$ & $\begin{array}{l}116.6 \\
(28.7)\end{array}$ & $\begin{array}{c}77.4 \\
(14.3)\end{array}$ \\
\hline 4) Sandy Point & $4.76 \times 10^{4}$ & $\begin{array}{c}35.1 \\
(12.8)\end{array}$ & $\begin{array}{c}32.6 \\
(10.0)\end{array}$ & $\begin{array}{c}64.0 \\
(14.0)\end{array}$ & $\begin{array}{c}25.1 \\
(9.4)\end{array}$ & $\begin{array}{l}11.6 \\
(4.0)\end{array}$ & $\begin{array}{l}13.6 \\
(4.7)\end{array}$ & $\begin{array}{l}133.1 \\
(20.7)\end{array}$ & $\begin{array}{l}135.6 \\
(27.2)\end{array}$ & $\begin{array}{c}83.1 \\
(31.4)\end{array}$ & $\begin{array}{c}58.6 \\
(9.2)\end{array}$ \\
\hline 5) Haynie Point & $1.92 \times 10^{4}$ & $\begin{array}{c}7.1 \\
(2.4)\end{array}$ & $\begin{array}{l}10.1 \\
(3.8)\end{array}$ & $\begin{array}{l}142.7 \\
(38.6)\end{array}$ & $\begin{array}{c}36.1 \\
(14.4)\end{array}$ & $\begin{array}{c}3.7 \\
(1.3)\end{array}$ & $\begin{array}{c}3.3 \\
(1.3)\end{array}$ & $\begin{array}{l}127.9 \\
(28.3)\end{array}$ & $\begin{array}{l}108.0 \\
(27.1)\end{array}$ & $\begin{array}{c}70.9 \\
(32.0)\end{array}$ & $\begin{array}{c}54.3 \\
(13.3)\end{array}$ \\
\hline 6) Fleet Point & $5.90 \times 10^{4}$ & $\begin{array}{c}8.9 \\
(3.3)\end{array}$ & $\begin{array}{l}14.9 \\
(4.0)\end{array}$ & $\begin{array}{c}26.3 \\
(7.7)\end{array}$ & $\begin{array}{c}8.4 \\
(5.2)\end{array}$ & $\begin{array}{c}0.9 \\
(0.5)\end{array}$ & $\begin{array}{c}0.1 \\
(0.1)\end{array}$ & $\begin{array}{c}4.6 \\
(1.4)\end{array}$ & $\begin{array}{c}25.9 \\
(7.5)\end{array}$ & $\begin{array}{c}12.4 \\
(7.4)\end{array}$ & $\begin{array}{c}15.0 \\
(8.0)\end{array}$ \\
\hline 7) Cranes Creek & $5.08 \times 10^{4}$ & $\begin{array}{l}13.6 \\
(2.2)\end{array}$ & $\begin{array}{l}11.1 \\
(2.1)\end{array}$ & $\begin{array}{l}12.1 \\
(4.3)\end{array}$ & $\begin{array}{c}3.7 \\
(1.1)\end{array}$ & $\begin{array}{l}1.0 \\
(0.6)\end{array}$ & $\begin{array}{c}1.3 \\
(0.8)\end{array}$ & $\begin{array}{c}40.3 \\
(11.3)\end{array}$ & $\begin{array}{c}67.1 \\
(17.1)\end{array}$ & $\begin{array}{c}61.9 \\
(15.8)\end{array}$ & $\begin{array}{c}31.7 \\
(12.2)\end{array}$ \\
\hline
\end{tabular}

Data are presented as number of oysters per square meter (SEM in parentheses); $n=7$ for all reef-year combinations. The numbers of the individual reefs correspond to those in Figure 1. 
TABLE 3.

Average oyster biomass (dry tissue weight in grams) on the Great Wicomico River public reefs from 2000 through 2009.

\begin{tabular}{|c|c|c|c|c|c|c|c|c|c|c|c|}
\hline $\begin{array}{c}\text { Station, } \\
\text { Reef Name }\end{array}$ & Area $\left(\mathrm{m}^{2}\right)$ & 2000 & 2001 & 2002 & 2003 & 2004 & 2005 & 2006 & 2007 & 2008 & 2009 \\
\hline 1) Rogue Point & $1.36 \times 10^{4}$ & $\begin{array}{l}12.4 \\
(5.8)\end{array}$ & $\begin{array}{l}1.8 \\
(1.0)\end{array}$ & $\begin{array}{l}22.4 \\
(6.7)\end{array}$ & $\begin{array}{c}5.2 \\
(1.6)\end{array}$ & $\begin{array}{l}7.8 \\
(3.3)\end{array}$ & $\begin{array}{c}2.9 \\
(1.3)\end{array}$ & $\begin{array}{l}68.8 \\
(8.1)\end{array}$ & $\begin{array}{c}82.5 \\
(13.9)\end{array}$ & $\begin{array}{l}80.8 \\
(9.4)\end{array}$ & $\begin{array}{l}21.5 \\
(6.2)\end{array}$ \\
\hline 2) Harcum Flats & $2.42 \times 10^{4}$ & $\begin{array}{l}11.5 \\
(5.5)\end{array}$ & $\begin{array}{c}6.3 \\
(1.8)\end{array}$ & $\begin{array}{l}12.9 \\
(2.3)\end{array}$ & $\begin{array}{l}15.7 \\
(6.2)\end{array}$ & $\begin{array}{l}10.8 \\
(5.1)\end{array}$ & $\begin{array}{c}2.7 \\
(1.2)\end{array}$ & $\begin{array}{l}17.0 \\
(4.4)\end{array}$ & $\begin{array}{c}87.6 \\
(17.4)\end{array}$ & $\begin{array}{c}99.9 \\
(20.1)\end{array}$ & $\begin{array}{c}89.9 \\
(10.4)\end{array}$ \\
\hline 3) Shell Bar & $7.16 \times 10^{4}$ & $\begin{array}{l}12.2 \\
(4.0)\end{array}$ & $\begin{array}{c}3.3 \\
(1.2)\end{array}$ & $\begin{array}{l}4.7 \\
(1.6)\end{array}$ & $\begin{array}{l}2.1 \\
(0.9)\end{array}$ & $\begin{array}{c}9.4 \\
(3.0)\end{array}$ & $\begin{array}{c}5.3 \\
(3.2)\end{array}$ & $\begin{array}{l}23.0 \\
(5.6)\end{array}$ & $\begin{array}{c}44.2 \\
(16.3)\end{array}$ & $\begin{array}{c}73.1 \\
(19.6)\end{array}$ & $\begin{array}{c}56.7 \\
(11.7)\end{array}$ \\
\hline 4) Sandy Point & $4.76 \times 10^{4}$ & $\begin{array}{c}8.9 \\
(3.8)\end{array}$ & $\begin{array}{l}6.8 \\
(2.6)\end{array}$ & $\begin{array}{c}5.7 \\
(1.2)\end{array}$ & $\begin{array}{c}6.7 \\
(2.8)\end{array}$ & $\begin{array}{c}9.6 \\
(4.1)\end{array}$ & $\begin{array}{l}12.0 \\
(4.5)\end{array}$ & $\begin{array}{l}17.0 \\
(6.1)\end{array}$ & $\begin{array}{c}49.0 \\
(11.3)\end{array}$ & $\begin{array}{c}51.1 \\
(18.8)\end{array}$ & $\begin{array}{c}38.3 \\
(7.5)\end{array}$ \\
\hline 5) Haynie Point & $1.92 \times 10^{4}$ & $\begin{array}{c}4.4 \\
(1.9)\end{array}$ & $\begin{array}{c}3.3 \\
(1.4)\end{array}$ & $\begin{array}{c}9.9 \\
(2.1)\end{array}$ & $\begin{array}{l}12.4 \\
(7.8)\end{array}$ & $\begin{array}{c}3.3 \\
(1.3)\end{array}$ & $\begin{array}{l}1.5 \\
(0.8)\end{array}$ & $\begin{array}{c}20.1 \\
(5.0)\end{array}$ & $\begin{array}{c}41.3 \\
(11.0)\end{array}$ & $\begin{array}{c}43.4 \\
(18.8)\end{array}$ & $\begin{array}{l}38.7 \\
(9.3)\end{array}$ \\
\hline 6) Fleet Point & $5.90 \times 10^{4}$ & $\begin{array}{c}2.9 \\
(1.3)\end{array}$ & $\begin{array}{c}6.1 \\
(2.0)\end{array}$ & $\begin{array}{c}5.0 \\
(1.9)\end{array}$ & $\begin{array}{c}5.5 \\
(3.4)\end{array}$ & $\begin{array}{c}0.5 \\
(0.3)\end{array}$ & $\begin{array}{c}0.01 \\
(0.01)\end{array}$ & $\begin{array}{c}0.4 \\
(0.2)\end{array}$ & $\begin{array}{c}6.9 \\
(2.4)\end{array}$ & $\begin{array}{c}3.4 \\
(1.9)\end{array}$ & $\begin{array}{l}14.5 \\
(8.7)\end{array}$ \\
\hline 7) Cranes Creek & $5.08 \times 10^{4}$ & $\begin{array}{c}4.0 \\
(0.6)\end{array}$ & $\begin{array}{l}2.8 \\
(0.7)\end{array}$ & $\begin{array}{l}1.9 \\
(1.1)\end{array}$ & $\begin{array}{l}1.3 \\
(0.6)\end{array}$ & $\begin{array}{c}0.6 \\
(0.5)\end{array}$ & $\begin{array}{l}0.2 \\
(0.1)\end{array}$ & $\begin{array}{c}3.0 \\
(0.8)\end{array}$ & $\begin{array}{c}22.1 \\
(5.8)\end{array}$ & $\begin{array}{l}20.7 \\
(5.4)\end{array}$ & $\begin{array}{c}20.2 \\
(7.3)\end{array}$ \\
\hline
\end{tabular}

Data are presented as biomass of oysters per square meter (SEM in parentheses); $n=7$ for all reef-year combinations.

ratios for live oyster data from the Great Wicomico River are between 1 and 60, with the remaining $13 \%$ between 60 and 150 (Fig. 4A), indicating an aggregated distribution. A considerable number of reefs exhibit ratio values of less than 20, whereas 7 reef-year combinations display ratios exceeding 60 . These are generally lower than the ratios of 10-100 reported for live oysters observed in the James River (Mann et al. 2009b) and the hard clam (Mercenaria mercenaria (Fegley 2001)).

The relationship between oyster SL (in millimeters) and oyster biomass (dry tissue weight in grams) for 172 oysters

TABLE 4.

Summary of statistics performed on the data from the Great Wicomico River public reefs.

\begin{tabular}{|c|c|c|c|c|c|c|c|}
\hline Test & Response & Factor & Covariate & df & $\mathrm{F} / \mathrm{T}$ value & $P$ value & $\begin{array}{l}\text { Multiple Comparison } \\
\text { Results (Fisher's test) }\end{array}$ \\
\hline \multirow[t]{3}{*}{ ANOVA } & Total shell volume & Location & & 1 & & $<0.01$ & Upriver $>$ downriver \\
\hline & & Year & & 9 & & $<0.01$ & $\begin{array}{l}2002,2009>2006, \\
2009>2004,2005\end{array}$ \\
\hline & & Location $\times$ year & & 9 & & $<0.01$ & \\
\hline \multirow[t]{4}{*}{ ANCOVA } & Oyster density & & Total volume & 1 & 142.96 & $<0.01$ & \\
\hline & & Location & & 1 & 139.28 & $<0.01$ & Upriver $>$ downriver \\
\hline & & Year & & 9 & 38.11 & $<0.01$ & $\begin{array}{c}2006>2002,2008 \\
2009>\text { all others } \\
2006=2007\end{array}$ \\
\hline & & Location $\times$ year & & 9 & 3.04 & $<0.01$ & \\
\hline \multirow[t]{4}{*}{ ANCOVA } & Oyster biomass & & Total volume & 1 & 107.72 & $<0.01$ & \\
\hline & & Location & & 1 & 97.78 & $<0.01$ & Upriver $>$ downriver \\
\hline & & Year & & & 29.24 & $<0.01$ & $\begin{array}{l}2007,2008,2009> \\
2006>\text { all others; } \\
2008>2009\end{array}$ \\
\hline & & Location $\times$ year & & 9 & 3.95 & $<0.01$ & \\
\hline \multirow[t]{4}{*}{ ANCOVA } & Oyster density & & Brown volume & 1 & 236.55 & $<0.01$ & \\
\hline & & Location & & 1 & 100.03 & $<0.01$ & Upriver $>$ downriver \\
\hline & & Year & & 7 & 55.45 & $<0.01$ & $\begin{array}{l}2002,2006,2007 \\
\quad 2008>2003,2004,2005\end{array}$ \\
\hline & & Location $\times$ year & & 7 & 2.07 & $<0.01$ & \\
\hline \multirow[t]{4}{*}{ ANCOVA } & Oyster biomass & & Brown volume & 1 & 110.27 & $<0.01$ & \\
\hline & & Location & & 1 & 63.83 & $<0.01$ & Upriver $>$ downriver \\
\hline & & Year & & 7 & 31.19 & $<0.01$ & $\begin{array}{r}2007,2008,2009>\text { all } \\
\text { others; } 2008>2009\end{array}$ \\
\hline & & Location $\times$ year & & 7 & 3.65 & $<0.01$ & \\
\hline
\end{tabular}

Significance levels were $p=0.05$. Upriver sites include reefs 1 through 5, downriver sites include reefs 6 and 7 (see Fig. 1 for exact locations within the system). 
TABLE 5.

Average total volume (in liters) of oyster shell in the Great Wicomico River from 2000 through 2009.

\begin{tabular}{|c|c|c|c|c|c|c|c|c|c|c|c|}
\hline $\begin{array}{c}\text { Station, } \\
\text { Reef Name }\end{array}$ & Area $\left(\mathrm{m}^{2}\right)$ & 2000 & 2001 & 2002 & 2003 & 2004 & 2005 & 2006 & 2007 & 2008 & 2009 \\
\hline 1) Rogue Point & $1.36 \times 10^{4}$ & $\begin{array}{l}10.0 \\
(3.3)\end{array}$ & $\begin{array}{c}6.4 \\
(2.6)\end{array}$ & $\begin{array}{l}16.9 \\
(3.4)\end{array}$ & $\begin{array}{l}10.5 \\
(5.1)\end{array}$ & $\begin{array}{c}9.2 \\
(2.4)\end{array}$ & $\begin{array}{l}7.6 \\
(2.0)\end{array}$ & $\begin{array}{l}16.9 \\
(2.4)\end{array}$ & $\begin{array}{l}15.9 \\
(1.9)\end{array}$ & $\begin{array}{l}17.7 \\
(1.6)\end{array}$ & $\begin{array}{c}8.9 \\
(1.6)\end{array}$ \\
\hline 2) Harcum Flats & $2.42 \times 10^{4}$ & $\begin{array}{l}12.0 \\
(3.9)\end{array}$ & $\begin{array}{l}22.6 \\
(3.0)\end{array}$ & $\begin{array}{l}20.9 \\
(3.3)\end{array}$ & $\begin{array}{l}13.9 \\
(4.1)\end{array}$ & $\begin{array}{l}11.7 \\
(3.2)\end{array}$ & $\begin{array}{l}18.8 \\
(3.7)\end{array}$ & $\begin{array}{c}9.4 \\
(2.3)\end{array}$ & $\begin{array}{l}18.9 \\
(3.4)\end{array}$ & $\begin{array}{l}18.8 \\
(1.9)\end{array}$ & $\begin{array}{l}27.1 \\
(1.4)\end{array}$ \\
\hline 3) Shell Bar & $7.16 \times 10^{4}$ & $\begin{array}{l}13.6 \\
(3.7)\end{array}$ & $\begin{array}{c}9.7 \\
(2.9)\end{array}$ & $\begin{array}{l}13.0 \\
(3.6)\end{array}$ & $\begin{array}{l}21.0 \\
(4.6)\end{array}$ & $\begin{array}{l}14.7 \\
(3.2)\end{array}$ & $\begin{array}{l}12.0 \\
(2.7)\end{array}$ & $\begin{array}{l}16.0 \\
(3.0)\end{array}$ & $\begin{array}{l}11.3 \\
(4.2)\end{array}$ & $\begin{array}{l}16.4 \\
(3.8)\end{array}$ & $\begin{array}{l}17.3 \\
(1.7)\end{array}$ \\
\hline 4) Sandy Point & $4.76 \times 10^{4}$ & $\begin{array}{l}10.7 \\
(3.7)\end{array}$ & $\begin{array}{l}12.1 \\
(3.2)\end{array}$ & $\begin{array}{l}14.6 \\
(2.8)\end{array}$ & $\begin{array}{l}12.9 \\
(3.8)\end{array}$ & $\begin{array}{l}11.4 \\
(2.6)\end{array}$ & $\begin{array}{l}11.1 \\
(2.2)\end{array}$ & $\begin{array}{l}13.0 \\
(1.6)\end{array}$ & $\begin{array}{l}15.7 \\
(3.1)\end{array}$ & $\begin{array}{l}11.1 \\
(3.7)\end{array}$ & $\begin{array}{l}20.0 \\
(2.5)\end{array}$ \\
\hline 5) Haynie Point & $1.92 \times 10^{4}$ & $\begin{array}{c}9.6 \\
(3.1)\end{array}$ & $\begin{array}{c}8.9 \\
(3.1)\end{array}$ & $\begin{array}{l}15.0 \\
(1.8)\end{array}$ & $\begin{array}{l}10.3 \\
(2.3)\end{array}$ & $\begin{array}{l}10.7 \\
(2.9)\end{array}$ & $\begin{array}{l}11.2 \\
(4.9)\end{array}$ & $\begin{array}{l}10.6 \\
(3.6)\end{array}$ & $\begin{array}{l}16.0 \\
(2.4)\end{array}$ & $\begin{array}{c}9.0 \\
(3.3)\end{array}$ & $\begin{array}{l}16.7 \\
(2.8)\end{array}$ \\
\hline 6) Fleet Point & $5.90 \times 10^{4}$ & $\begin{array}{c}6.7 \\
(2.1)\end{array}$ & $\begin{array}{l}13.0 \\
(2.7)\end{array}$ & $\begin{array}{c}8.9 \\
(2.6)\end{array}$ & $\begin{array}{c}4.9 \\
(2.5)\end{array}$ & $\begin{array}{c}6.5 \\
(2.8)\end{array}$ & $\begin{array}{l}1.3 \\
(0.6)\end{array}$ & $\begin{array}{c}3.5 \\
(1.3)\end{array}$ & $\begin{array}{c}7.8 \\
(1.9)\end{array}$ & $\begin{array}{c}4.3 \\
(2.1)\end{array}$ & $\begin{array}{c}5.6 \\
(2.3)\end{array}$ \\
\hline 7) Cranes Creek & $5.08 \times 10^{4}$ & $\begin{array}{l}16.3 \\
(1.0)\end{array}$ & $\begin{array}{l}12.0 \\
(1.1)\end{array}$ & $\begin{array}{l}16.2 \\
(3.4)\end{array}$ & $\begin{array}{l}12.9 \\
(1.7)\end{array}$ & $\begin{array}{c}7.4 \\
(3.1)\end{array}$ & $\begin{array}{c}6.8 \\
(1.2)\end{array}$ & $\begin{array}{c}9.1 \\
(1.7)\end{array}$ & $\begin{array}{l}12.0 \\
(2.6)\end{array}$ & $\begin{array}{l}12.0 \\
(3.0)\end{array}$ & $\begin{array}{l}18.2 \\
(1.4)\end{array}$ \\
\hline
\end{tabular}

Data are presented as total shell volume per square meter (SEM in parentheses); $n=7$ for all reef-year combinations.

ranging from 16.5-98.5 $\mathrm{mm}$ SL was described using a power equation:

$$
\text { Biomass }=9.6318 \times 10^{-6} \times \mathrm{SL}^{(2.743)} ; \quad R^{2}=0.64
$$

Year- and reef-specific demographic data were used to generate a description of reef-specific oyster biomass (measured in grams of dry tissue) for 2000 through 2008 as summarized in Table 3. The grouping of reefs previously described for density (Table 2) also applies for biomass.

Biomass on upriver reefs was generally less than $13 \mathrm{~g} / \mathrm{m}^{2}$ for 2000 through 2005, with the exceptions of reef 1 in 2002 and reef 2 in 2003 (Table 3). These same reefs exhibited mean biomass values in the range $17.0-99.9 \mathrm{~g} / \mathrm{m}^{2}$ for 2006 through 2009 (Table 3). Downriver reefs had significantly lower biomass than the upriver reefs (ANCOVA, Table 4). In general, these were consistently less than or equal to $6 \mathrm{~g} / \mathrm{m}^{2}$, with the exception of reef 7 in 2007 through 2009 at 22.1, 20.7, and $20.2 \mathrm{~g} / \mathrm{m}^{2}$, respectively. Biomass increased at the upriver reefs in 2006 through 2008 despite the decreasing densities. This trend reflects growth of the surviving oysters from the 2006 recruitment and mortality of larger oysters at those same reefs in 2009. There was a significant interaction between location and year for both density and biomass (ANCOVA, Table 4).

\section{The Shell Resource}

Shell material collected in the surveys and quantified in units of liters per square meter for each sample ranged in size from intact oyster shells to shell fragments. The recorded reef and yearspecific values of total shell (brown plus black) and brown shell for the study period are given in Table 5 (2000 through 2009) and Table 6 (2002 through 2009), respectively. Brown shell results from mortality. The time frame for its decay or disappearance may be on the order of years (Powell et al. 2006). Black shell results from burial of brown shell, and the decay or disappearance rate may then change. In addition to shell from natural recruitment, growth, and mortality processes, selected reefs received supplemental shell planting at various junctures during the study period (Table 7). These values are reflected in Tables 5 and 6 , although shell additions must be considered in the context of shell removal as part of seed harvesting for the purposes of a shell budget (described later). Shell planting and seed harvest activities mask the natural burial and degradation processes.

Total shell volume was significantly higher in upriver reefs than downriver reefs (Table 4). The upriver reefs varied from 9.7-27.1 L/m $\mathrm{m}^{2}$, with most values in the $11.0-20.0-\mathrm{L} / \mathrm{m}^{2}$ range. Downriver reefs varied from $1.3-16.3 \mathrm{~L} / \mathrm{m}^{2}$, with all but 4 records being less than or equal to $12 \mathrm{~L} / \mathrm{m}^{2}$. Brown shell volume on the upriver reefs varied between $2.0 \mathrm{~L} / \mathrm{m}^{2}$ at reef 3 in 2002 and $18.2 \mathrm{~L} / \mathrm{m}^{2}$ at reef 1 in 2006 . The relationship of total and brown shell to shell addition as part of repletion activity (Table 7) illustrate the impoverished state of the underlying shell substrate, possibly the result of many years of sustained disease pressure during 1970 through 1990 (Andrews 1996, Burreson \& Ragone Calvo 1996), shell decay, and the sinking of added shell below the sediment-water interface in modest time periods.

The variance-to-mean ratio values for both total and brown shell (Fig. 4B and C) are consistently less than 20 and mostly less than 10. The pattern appears to be independent of oyster density and total shell volume (compare Fig. 4A and B). These values indicate aggregation in the shell resource with space and time, which is to be expected from an animal with gregarious settlement patterns that creates a biogenic habitat.

\section{Relationship of Live Oyster Density, Biomass, and Shell Volume over Time}

The spatial variation in absolute density reflects cumulative patterns of recruitment and postrecruit survival. Biomass-perunit-area values are less influenced by sporadic recruitment events, because the losses in number after recruitment are offset by the increasing biomass per individual. An increase in biomass is expected in the 1-2 y following a year of good recruitment, as was observed between 2002 (high density) and 2003 through 2005 (high biomass), and 2006 (high density) and 2007 through 2008 (high biomass), although a general decrease 
in biomass was observed at all reefs except reef 6 in 2009. Mann et al. (2009b) note that increasing recruitment and overall oyster density are often associated with higher salinity in the James River. Mid 2002 was the highest salinity period during the current observation period (Fig. 3), with weekly records exceeding 22 ppt. However, in 2006, salinities never exceeded $16 \mathrm{ppt}$, lower than values observed in the summers of 2000 through 2002, 2005, and 2007 through 2008. Extended low salinities were observed through the summer of 2003. Salinities in winter and spring 2009 remained above 14 ppt (Fig. 3).

The relationship between oyster SL (in millimeters) and oyster wet shell weight (in grams) for 398 Great Wicomico River oysters (SL range, 16.5-115.6 mm) collected between 2004 and 2007 was described using a power equation:

$$
\begin{aligned}
\text { Wet shell weight } & =0.00017324 \times \text { Shell length }^{(2.9926)} ; \\
R^{2} & =0.79
\end{aligned}
$$

This relationship was used to estimate the amount of live shell (measured in grams) observed in each patent tong on the basis of the available live oysters. The volumetric conversion from Mann et al. (2009b) was used to convert grams of shell to liters of shell herein.

\section{Oyster Standing Stocks}

Standing stock in numbers and biomass was estimated by multiplying the average oyster density and biomass per unit area (Tables 2 and 3) by the reef area. Table 8 shows year- and reef-specific as well as the total (sum of all reefs) estimated standing stock for 2000 through 2009. Table 9 gives the corresponding biomass. Total numbers of oysters in the surveyed area varied between a low value of $1.56 \times 10^{6}$ in 2005 and a high value of $3.63 \times 10^{7}$ in 2006 . A recruitment event in 2002 increased the standing stock 4-fold over 2001, with an increase from $5.56 \times 10^{6}$ to $2.11 \times 10^{7}$ in 2002 . A total of 18,583 bushels at 500 per bushel count of seed were removed from the upriver reefs, mostly reefs $3-5$. Thus, an estimated $0.93 \times 10^{7}$ oysters were removed from the upriver reefs in 2003. A comparison of the estimated 2003 seed removal indicates that approximately half the difference that we see in the 2002 versus 2003 upriver standing stock estimate for the upriver reefs is the result of the seed harvest. The decreasing trend in standing stock continued through 2005 with a value of $1.56 \times 10^{6}$, or a 13 -fold reduction over a 3 -y period. The recruitment event in 2006 was a 23 -fold increase in standing stock compared with 2005. A decrease in standing stock to $1.61 \times 10^{7}$ was recorded through 2009 .

Biomass decreased from $2000\left(2.2 \times 10^{4} \mathrm{~g}\right)$ to the 2001 value of $1.30 \times 10^{6} \mathrm{~g}$. The 2002 recruitment event resulted in a biomass increase to $1.82 \times 10^{6} \mathrm{~g}$, followed by a decrease to a 2005 value of $1.09 \times 10^{6} \mathrm{~g}$. Biomass increased in 2006 commensurate with strong recruitment. The 2006 through 2008 period was accompanied by a 12 -fold increase in biomass to a 2008 value of $1.33 \times$ $10^{7} \mathrm{~g}$.

\section{Estimation of Oyster Age at Length}

The quadratic relationship SL $=a \times(\text { Age })^{2}+b \times($ Age $)+c$ was used to estimate age at length for Great Wicomico River oysters (Fig. 5). Values for $a, b$, and $c$ for 2003 through 2009 are given in Table 10 . Using a July 1 birth date and noting that current data are for a fall survey, then lengths on November 1
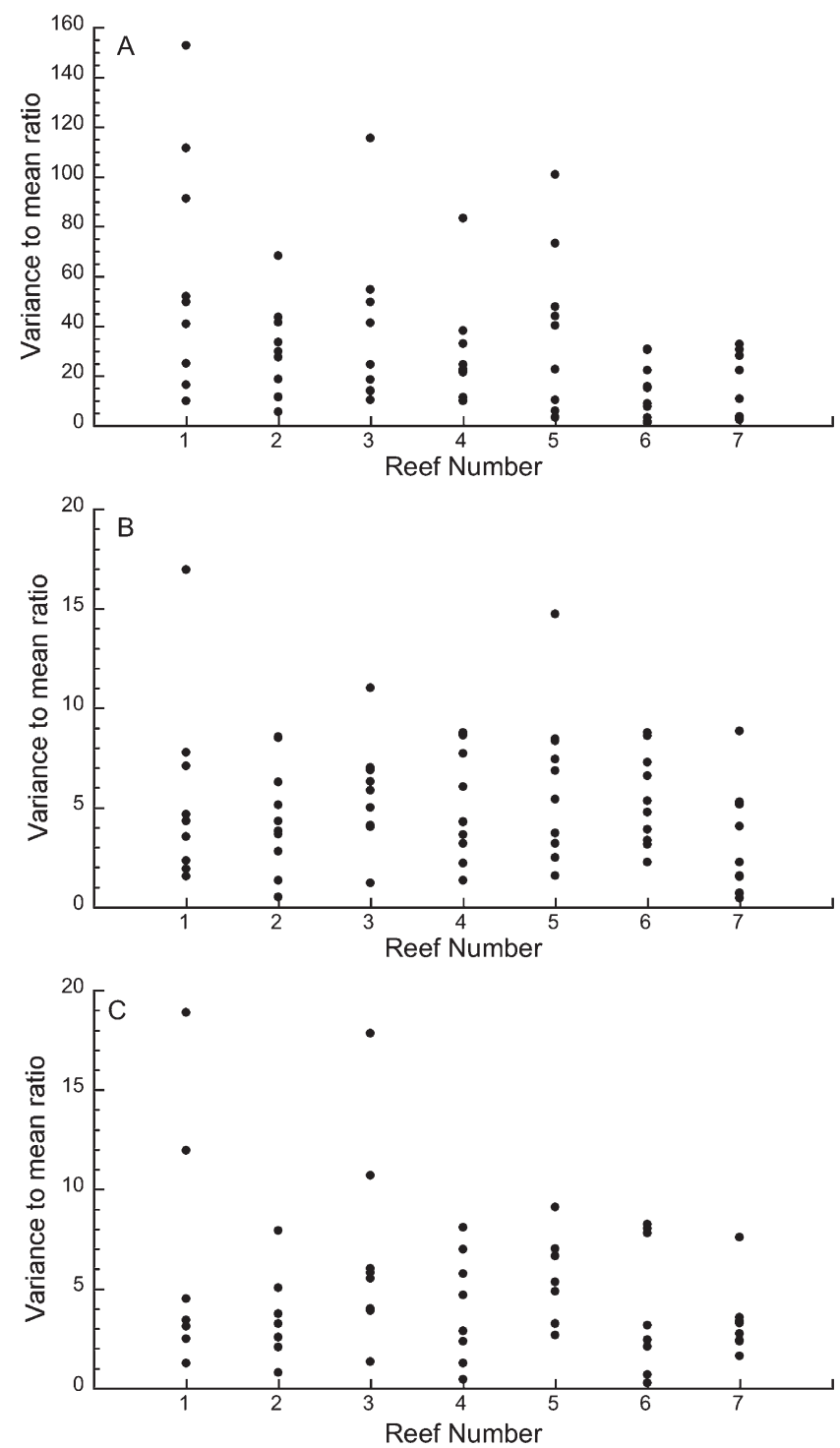

Figure 4. (A-C) Variance to mean ratios of oyster density (A; 2000 through 2009), total shell volume (B; 2000 through 2009), and brown shell volume $(C ; 2002$ through 2009) for the seven public reefs in the Great Wicomico River. Note the difference in scale for the $y$-axis in panels $B$ and $C$ (20) relative to the scale on the y-axis in panel A (160).

represent ages of $0.33 \mathrm{y}, 1.33 \mathrm{y}, 2.33 \mathrm{y}$ and so on with annual increments. For clarity throughout the rest of the text, these ages will be referred to as Age 0, 1, 2, 3, and 4, respectively. As an example, corresponding lengths for 2003 are $17.5 \mathrm{~mm}$ at $0 \mathrm{y}$, $52.1 \mathrm{~mm}$ at $1 \mathrm{y}, 75.4 \mathrm{~mm}$ at $2 \mathrm{y}$, and $87.4 \mathrm{~mm}$ at $3 \mathrm{y}$. Examination of year-specific data revealed very modest changes in the $a, b$, and $c$ values from year to year (Table 10). The age-at-length model was used to recast the length demographic as an age demographic and to estimate age-specific mortality as described earlier.

The current estimates of oyster age at length are commensurate with 0 to 3 -y classes for the linear age-at-length relationships reported by Harding et al. (2008) and Mann et al. (2009b) for oyster populations in the James River, VA. The curvature of the quadratic fit dictates lower length at age for older 
TABLE 6.

Average volume (in liters) of brown oyster shell in the Great Wicomico River from 2000 through 2009.

\begin{tabular}{|c|c|c|c|c|c|c|c|c|c|}
\hline $\begin{array}{c}\text { Station, } \\
\text { Reef Name }\end{array}$ & Area $\left(m^{2}\right)$ & 2002 & 2003 & 2004 & 2005 & 2006 & 2007 & 2008 & 2009 \\
\hline 1) Rogue Point & $1.36 \times 10^{4}$ & $\begin{array}{l}12.1 \\
(1.9)\end{array}$ & $\begin{array}{l}9.0 \\
(0.9)\end{array}$ & $\begin{array}{c}8.4 \\
(0.7)\end{array}$ & $\begin{array}{c}6.7 \\
(0.9)\end{array}$ & $\begin{array}{l}16.6 \\
(0.6)\end{array}$ & $\begin{array}{l}14.0 \\
(0.3)\end{array}$ & $\begin{array}{l}15.1 \\
(0.8)\end{array}$ & $\begin{array}{c}7.0 \\
(0.7)\end{array}$ \\
\hline 2) Harcum Flats & $2.42 \times 10^{4}$ & ND & $\begin{array}{c}8.6 \\
(1.2)\end{array}$ & $\begin{array}{c}8.0 \\
(0.9)\end{array}$ & $\begin{array}{l}11.1 \\
(0.9)\end{array}$ & $\begin{array}{l}7.0 \\
(0.7)\end{array}$ & $\begin{array}{l}15.4 \\
(0.9)\end{array}$ & $\begin{array}{l}15.4 \\
(0.8)\end{array}$ & $\begin{array}{l}17.6 \\
(0.5)\end{array}$ \\
\hline 3) Shell Bar & $7.16 \times 10^{4}$ & $\begin{array}{c}2.0 \\
(0.5)\end{array}$ & $\begin{array}{l}12.4 \\
(2.1)\end{array}$ & $\begin{array}{l}10.9 \\
(0.9)\end{array}$ & $\begin{array}{c}6.6 \\
(0.9)\end{array}$ & $\begin{array}{l}11.7 \\
(1.0)\end{array}$ & $\begin{array}{c}8.0 \\
(1.3)\end{array}$ & $\begin{array}{l}12.5 \\
(1.2)\end{array}$ & $\begin{array}{l}10.9 \\
(0.5)\end{array}$ \\
\hline 4) Sandy Point & $4.76 \times 10^{4}$ & $\begin{array}{c}2.8 \\
(0.3)\end{array}$ & $\begin{array}{l}7.8 \\
(1.1)\end{array}$ & $\begin{array}{l}7.1 \\
(0.9)\end{array}$ & $\begin{array}{l}7.3 \\
(0.7)\end{array}$ & $\begin{array}{c}6.1 \\
(0.5)\end{array}$ & $\begin{array}{l}11.1 \\
(1.0)\end{array}$ & $\begin{array}{l}7.7 \\
(1.0)\end{array}$ & $\begin{array}{l}8.7 \\
(0.3)\end{array}$ \\
\hline 5) Haynie Point & $1.92 \times 10^{4}$ & ND & $\begin{array}{c}6.8 \\
(1.0)\end{array}$ & $\begin{array}{l}7.5 \\
(0.9)\end{array}$ & $\begin{array}{c}3.6 \\
(0.4)\end{array}$ & $\begin{array}{c}7.9 \\
(1.2)\end{array}$ & $\begin{array}{c}8.4 \\
(1.0)\end{array}$ & $\begin{array}{c}6.5 \\
(1.0)\end{array}$ & $\begin{array}{l}7.8 \\
(0.7)\end{array}$ \\
\hline 6) Fleet Point & $5.90 \times 10^{4}$ & $\begin{array}{c}4.1 \\
(0.5)\end{array}$ & $\begin{array}{l}1.4 \\
(0.3)\end{array}$ & $\begin{array}{l}2.3 \\
(0.6)\end{array}$ & $\begin{array}{c}0.4 \\
(0.1)\end{array}$ & $\begin{array}{l}1.0 \\
(0.1)\end{array}$ & $\begin{array}{c}2.5 \\
(0.3)\end{array}$ & $\begin{array}{l}2.8 \\
(0.7)\end{array}$ & $\begin{array}{c}3.6 \\
(0.8)\end{array}$ \\
\hline 7) Cranes Creek & $5.08 \times 10^{4}$ & $\begin{array}{l}3.0 \\
(0.3)\end{array}$ & $\begin{array}{l}3.1 \\
(0.4)\end{array}$ & $\begin{array}{l}2.8 \\
(0.4)\end{array}$ & $\begin{array}{c}2.5 \\
(0.4)\end{array}$ & $\begin{array}{c}3.7 \\
(0.5)\end{array}$ & $\begin{array}{c}5.0 \\
(0.6)\end{array}$ & $\begin{array}{c}9.4 \\
(1.2)\end{array}$ & $\begin{array}{r}6.2 \\
(0.7)\end{array}$ \\
\hline
\end{tabular}

Data are presented as brown shell volume per square meter (SEM in parentheses); $n=7$ for all reef-year combinations. ND, data not available for a particular site/year.

individuals than a linear fit. The rates reported herein are generally higher than those reported in Kraeuter et al. (2007) for natural oyster populations in Delaware Bay and growth rate estimates by Mann \& Evans (2004) for James River oyster populations.

\section{Age Structure, Mortality, and Recruit-to-Stock Ratio}

Age structure and mortality for the upriver and downriver reefs are presented in Table 11. Moving diagonally across and down, Table 11 follows the progression of a cohort over time. Note the harvest of seed oysters prior to the 2003 survey. Mortality was estimated as described in Eq. 1, omitting estimates for 2002 through 2003, which are confounded by seed removal (discussed earlier). Estimates of age-specific mortality by year class are compromised by very low densities, possible assignment of individuals to incorrect class (see Mann et al. (2009b) for a discussion), and the corresponding large apparent errors that result from wide differences in densities in successive years (e.g., 2005 and 2006). Thus, mortality estimates where both the \#Live $(t)$ and the \#Live $(t+1)$ values in Eq. 1 exceed 10/ $\mathrm{m}^{2}$ are distinguished in Table 11 .

Mortality at all reefs was generally high, as illustrated by a comparison between the 0 - and 1-y age values within a cohort (Table 11). High recruitment in 2002 and 2006 did not result in a corresponding high density of 3-y-olds observed in 2005 and 2009. Four-year-olds were absent throughout the system in all years examined. During 2006 through 2008, the 2006-y class suffered a cumulative total of 0.88 proportional mortality on upriver reefs and 0.87 on downriver reefs. It is important to reiterate that the young-of-the-year (YOY) value is for recruits surviving to the fall survey, and the generated proportions are from November through November. The values do not include presumed high mortality between metamorphosis and the initial fall survey. Mortality between YOY and year 2 for the 2007-y class is estimated at 0.62 and 0.71 for upriver and downriver reefs, respectively. No estimates of mortality were made by traditional comparisons of live animals to articulated valves,

TABLE 7.

Shell additions ("planting," measured in liters per square meter) from 2000 through 2009 to the seven public reefs in the Great Wicomico River.

\begin{tabular}{|c|c|c|c|c|c|c|c|c|c|c|c|}
\hline $\begin{array}{c}\text { Station, } \\
\text { Reef Name }\end{array}$ & Area $\left(\mathrm{m}^{2}\right)$ & 2000 & 2001 & 2002 & 2003 & 2004 & 2005 & 2006 & 2007 & 2008 & 2009 \\
\hline 1) Rogue Point & $1.36 \times 10^{4}$ & 8.2 & 17.2 & 0 & 16.5 & 16.6 & 0 & 11.3 & 10.9 & 0 & 0 \\
\hline 2) Harcum Flats & $2.42 \times 10^{4}$ & 10.0 & 18.3 & 0 & 30.5 & 19.3 & 0 & 0 & 12.2 & 0 & 0 \\
\hline 3) Shell Bar & $7.16 \times 10^{4}$ & 4.4 & 0 & 0 & 20.5 & 4.8 & 5.6 & 7.0 & 4.1 & 0 & 0 \\
\hline 4) Sandy Point & $4.76 \times 10^{4}$ & 4.7 & 8.4 & 0 & 9.0 & 4.6 & 0 & 0 & 3.1 & 0 & 0 \\
\hline 5) Haynie Point & $1.92 \times 10^{4}$ & 0 & 0 & 8.9 & 0 & 11.4 & 0 & 0 & 0 & 0 & 0 \\
\hline 6) Fleet Point & $5.90 \times 10^{4}$ & 0 & 0 & 0 & 0 & 0 & 0 & 0 & 0 & 32.8 & 18.6 \\
\hline 7) Cranes Creek & $5.08 \times 10^{4}$ & 0 & 0 & 0 & 0 & 0 & 0 & 0 & 0 & 28.3 & 0 \\
\hline
\end{tabular}

0 , no shell added to that particular reef during that year. 

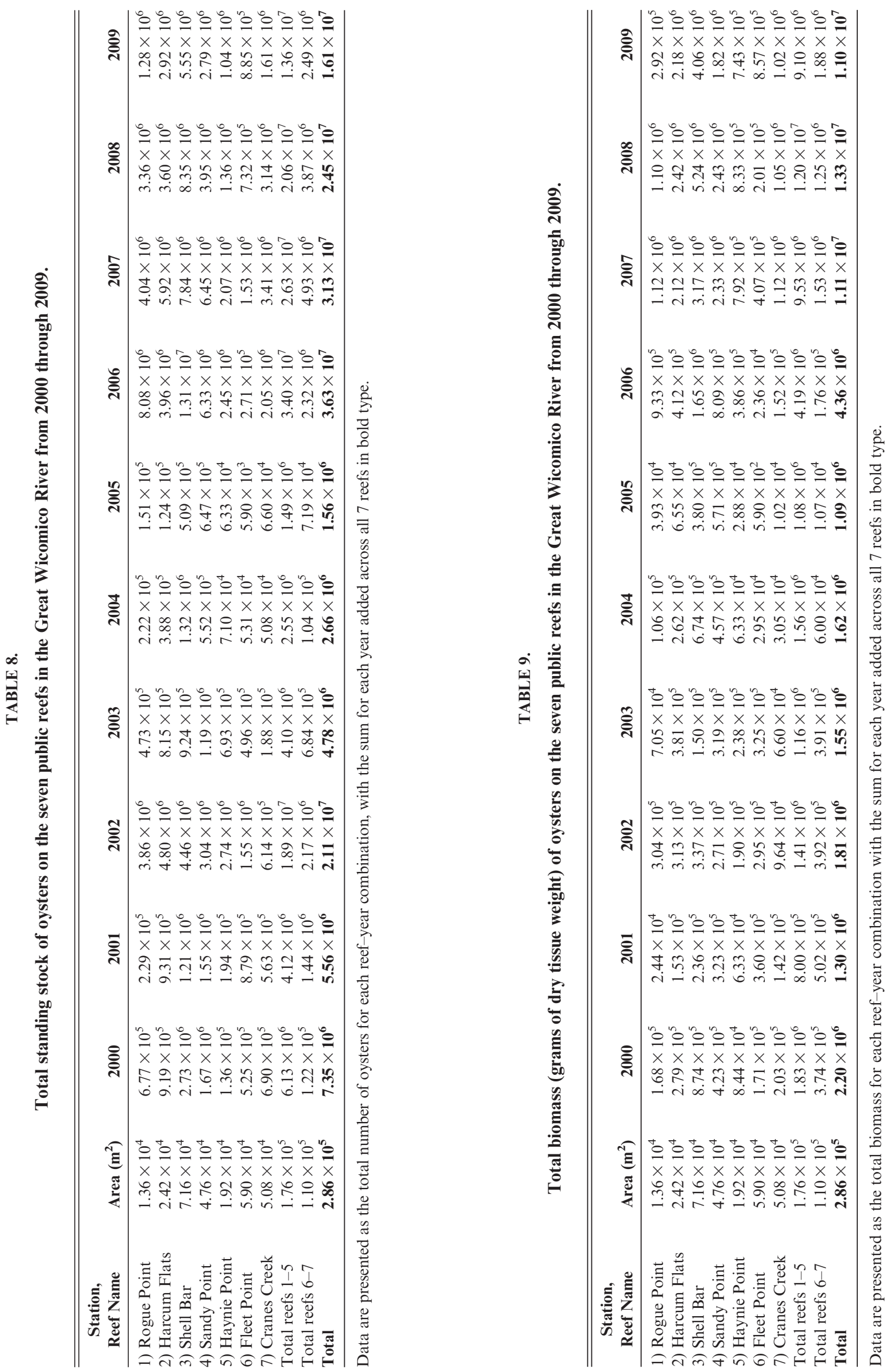


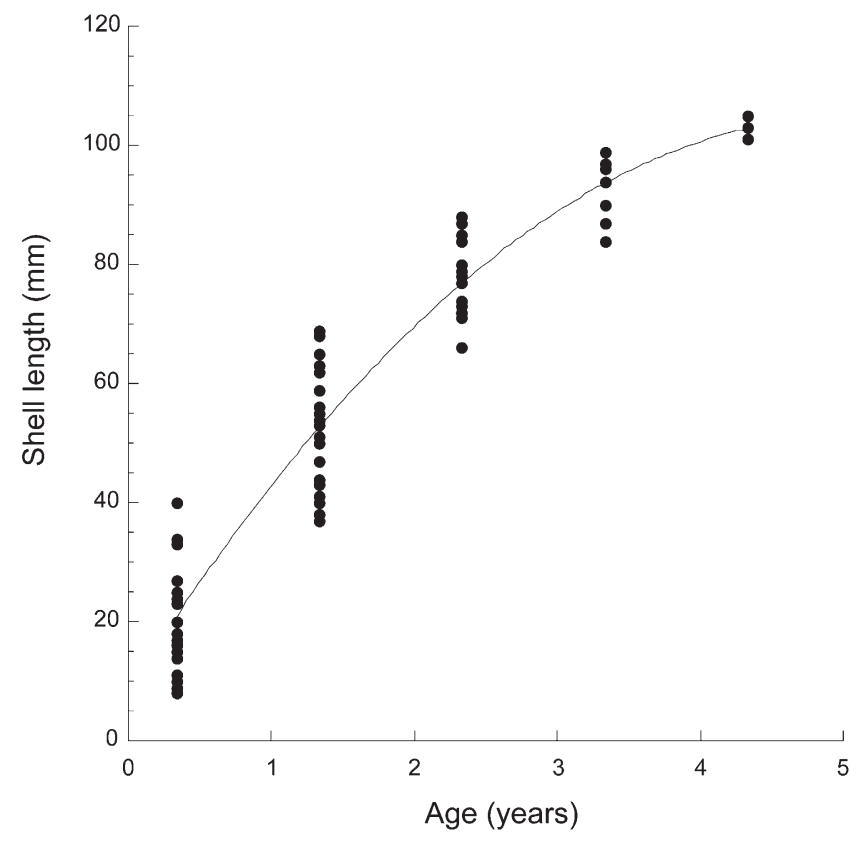

Figure 5. Estimation of age at length based on data from all reefs from 2003 through 2009. The quadratic model is $\mathrm{SL}=-3.7603 \times(\text { Age })^{2}+$ 38.0773 $\times($ Age $)+8.5144 ; R^{2}=0.92$.

commonly termed "box count methods," in that these have been shown (Mann et al. 2009b) to underestimate mortality based on demographics.

Recruitment in 2002 was almost an order of magnitude higher at the upriver reefs $\left(94.1 / \mathrm{m}^{2}\right)$ than downriver $\left(14.2 / \mathrm{m}^{2}\right)$. The highest recruitment values during the study were observed from 2006 through 2009, with upriver reef values of 176.4, 53.2, 31.9 , and $32.9 / \mathrm{m}^{2}$, respectively; and downriver reef values of $19.9,22.5,19.5$, and $6.4 / \mathrm{m}^{2}$, respectively. Note the gradual decline in recruitment at the upriver reefs from 2006 through 2008; recruitment at the upriver reefs was $\sim 32$ in 2008 as in 2009. Recruitment was essentially absent in 2004 and 2005.

The separation of the YOY from $\geq 1$-y-old oysters is based on the age-at-length relationship in Figure 5 and Table 10. The recruit-to-stock (R:S) ratio is generated from the stock (S, year $t)$ and recruit $(\mathrm{R}$, year $t+1)$ data. This ratio describes the riverwide stock at basinwide spatial scales, with an assumption of no immigration, and where all larvae have the opportunity to recruit within the basin. We explore this ratio for upriver and downriver reefs as separate regions, and a summation of all reefs combined. We cannot identify a predictive $\mathrm{R}: \mathrm{S}$ relationship for any subset of reefs or the total population on all reefs. Considering just the upriver reefs, the R:S ratio exceeded 1.0 in 2002, 2006, and 2007 (Table 11). Considering all reefs together, the R:S ratio exceeded 1.0 in 2002, 2006, and 2007, with values of 10.0, 31.0, and 4.0, respectively. The 2003 signal was compromised by seed removal from the upriver reefs. The R:S "replacement ratio" of more than 1.0 would typically suggest an expanding population over time. However, the high mortalities in age classes $1+$, discussed earlier in the text, result in a corresponding stock size (in absolute numbers) less than that of a single YOY cohort. The $2006 \mathrm{R}: \mathrm{S}$ value dominates all other observed values (Table 11) and was observed with a single recruitment event of $3.33 \times 10^{7}$ YOY (Table 11, YOY in 2006 estimated from total standing stock (Table 8) for all reefs combined) concurrent with the second lowest standing stock, $1.08 \times 10^{6}$ (all reefs, 2005) during the entire study.

\section{Disease Impacts on Mortality}

Dermo ( $P$. marinus) and MSX (H. nelsoni) have been recorded in the Great Wicomico River (Fig. 6) since the 1960s (Andrews \& Wood 1967, Andrews 1988). The prevalence and intensity of both diseases are related to temperature and salinity (Ford \& Haskin 1982, Andrews 1988). Winter temperatures in excess of $5^{\circ} \mathrm{C}$ are considered "warm" in the Chesapeake region (Andrews 1988, Mann et al. 2009b) and may foster Dermo development (Burreson \& Ragone Calvo 1996). Water temperatures greater than $5^{\circ} \mathrm{C}$ were observed in the Great Wicomico during 2001 to 2002, 2005 to 2006, and 2007 to 2008 with higher proportional mortalities (Table 11) and higher Dermo WP values (Fig. 6B) observed the following October.

Dermo can remain as very low-level infections during winter periods with salinities greater than $3 \mathrm{ppt}$, whereas MSX is expulsed at salinities less than 10 ppt (Burreson \& Ragone Calvo 1996). The presence of either disease when winter salinities are high $(>12-15 \mathrm{ppt})$ can accelerate epizootics in the following spring and summer. Overwintering salinity conditions observed

TABLE 10.

Regression coefficients for the Great Wicomico public reef oyster age-at-length relationships using the quadratic model $\mathrm{SL}=a \times(\text { Age })^{2}+b \times($ Age $)+c$.

\begin{tabular}{lccccrrr}
\hline \hline Year & $\mathbf{N}^{*}$ & $\boldsymbol{a}$ & $\boldsymbol{a}$ SE & $\boldsymbol{b}$ & $\boldsymbol{b}$ SE & $\boldsymbol{c}$ & $\boldsymbol{c}$ SE \\
\hline All & 84 & -3.7603 & 0.6267 & 38.0773 & 2.5982 & 8.5144 & 2.1578 \\
2003 & 10 & -5.6211 & 2.8875 & 43.8723 & 10.0736 & 3.6600 & 7.2440 \\
2004 & 11 & -6.3164 & 2.0677 & 45.8915 & 9.1012 & 1.8796 & 6.1287 \\
2005 & 8 & -3.1667 & 7.0553 & 32.5900 & 17.4640 & 13.2568 & 9.2202 \\
2006 & 13 & -4.8741 & 2.5047 & 42.6371 & 9.1526 & 7.8293 & 6.5873 \\
2007 & 12 & -4.1157 & 1.8417 & 41.2560 & 8.2164 & 5.0477 & 7.5229 \\
2008 & 12 & -3.8797 & 1.6235 & 39.7282 & 7.2429 & 5.8302 & 6.6316 \\
2009 & 17 & -3.2049 & 1.1398 & 34.2472 & 5.0287 & 13.8610 & 4.2250 \\
\hline
\end{tabular}

$* \mathrm{~N}=$ number of (Age, SL) data pairs from cohort analysis that contribute to each equation.

Cohort analyses (Bhattacharya 1967) were conducted on individual oyster measurements ( $n=22,253$ oysters) made from 2003 through 2009. 
TABLE 11.

Age structure, age-specific mortality, and recruit-to-stock ratios on the seven public reefs examined in the Great Wicomico River for 2000 through 2009.

\begin{tabular}{|c|c|c|c|c|c|c|c|c|c|c|c|c|}
\hline Area & Size $\left(m^{2}\right)$ & Age & 2000 & 2001 & 2002 & 2003 & 2004 & 2005 & 2006 & 2007 & 2008 & 2009 \\
\hline \multicolumn{13}{|c|}{ Density:Oyster $\mathrm{m}^{-2}$} \\
\hline \multirow[t]{6}{*}{ Upriver } & $1.75 \times 10^{5}$ & YOY & 10.0 & 15.9 & 94.1 & 10.2 & 0.8 & 2.4 & 176.4 & 53.2 & 31.9 & 32.9 \\
\hline & & 1 & 23.9 & 6.8 & 12.5 & 12.3 & 9.9 & 3.7 & 13.2 & 91.5 & 62.2 & 20.6 \\
\hline & & 2 & 0.7 & 0.5 & 0.5 & 0.6 & 3.6 & 2.4 & 2.4 & 4.2 & 21.8 & 20.0 \\
\hline & & 3 & 0.1 & 0.1 & 0.0 & 0.2 & 0.2 & 0.0 & 0.5 & 0.2 & 0.8 & 2.3 \\
\hline & & 4 & 0.0 & 0.0 & 0.0 & 0.0 & 0.0 & 0.0 & 0.0 & 0.3 & 0.3 & 1.2 \\
\hline & & Total & 34.7 & 23.3 & 107.1 & 23.3 & 14.5 & 8.5 & 192.5 & 149.4 & 117.0 & 77.0 \\
\hline \multirow[t]{6}{*}{ Downriver } & $1.10 \times 10^{5}$ & YOY & 3.0 & 8.4 & 14.2 & 1.6 & 0.1 & 0.6 & 19.9 & 22.5 & 19.5 & 6.4 \\
\hline & & 1 & 7.7 & 3.2 & 4.8 & 3.6 & 0.4 & 0.1 & 1.2 & 20.6 & 12.1 & 10.6 \\
\hline & & 2 & 0.3 & 1.2 & 0.5 & 0.5 & 0.2 & 0.0 & 0.0 & 1.0 & 2.6 & 6.6 \\
\hline & & 3 & 0.0 & 0.3 & 0.1 & 0.5 & 0.0 & 0.0 & 0.0 & 0.0 & 0.1 & 1.0 \\
\hline & & 4 & 0.0 & 0.1 & 0.0 & 0.0 & 0.0 & 0.0 & 0.0 & 0.0 & 0.1 & 0.1 \\
\hline & & Total & 11.0 & 13.1 & 19.7 & 6.3 & 0.7 & 0.7 & 21.1 & 44.2 & 34.7 & 24.5 \\
\hline \multicolumn{13}{|c|}{ Age-Specific Mortality } \\
\hline \multirow[t]{4}{*}{ Upriver } & & 1 & & 0.3 & 0.2 & & 0.0 & -3.6 & -4.4 & 0.5 & -0.2 & 0.4 \\
\hline & & 2 & & 1.0 & 0.9 & & 0.7 & 0.8 & 0.4 & 0.7 & 0.8 & 0.7 \\
\hline & & 3 & & 0.9 & 1.0 & & 0.6 & 1.0 & 0.8 & 0.9 & 0.8 & 0.9 \\
\hline & & 4 & & 1.0 & 1.0 & & 1.0 & 1.0 & & 0.5 & -0.5 & -0.4 \\
\hline \multirow[t]{4}{*}{ Downriver } & & 1 & & -0.1 & 0.4 & & 0.7 & 0.3 & -1.1 & 0.0 & 0.5 & 0.5 \\
\hline & & 2 & & 0.9 & 0.8 & & 1.0 & 1.0 & 1.0 & 0.2 & 0.9 & 0.5 \\
\hline & & 3 & & 0.1 & 0.9 & & 0.9 & 1.0 & & & 0.9 & 0.6 \\
\hline & & 4 & & & & & & & & & & \\
\hline \multicolumn{13}{|c|}{ Recruit-to-Stock Ratio } \\
\hline Upriver & & & & 0.6 & 12.8 & 0.8 & 0.1 & 0.2 & 29.0 & 3.3 & 0.3 & 0.4 \\
\hline Downriver & & & & 1.1 & 3.0 & 0.3 & 0.0 & 0.9 & 375.8 & 18.4 & 0.9 & 0.4 \\
\hline All reefs & & & & 0.7 & 10.0 & 0.7 & 0.1 & 0.2 & 30.9 & 4.0 & 0.4 & 0.4 \\
\hline
\end{tabular}

Note that seed oyster removal occurred prior to the survey in 2003, thus values for 2003 are in bold and italic, and no estimates of age-specific mortality are offered for 2002 through 2003. All values of age-specific mortality estimated from densities of less than 10 oysters $/ \mathrm{m}^{2}$ are presented in italics.

in the Great Wicomico during 2002 to 2003,2003 to 2004 , and 2004 to 2005 were less than 10 ppt (Fig. 3), supporting expulsion of MSX.

With the exception of 2000, Dermo WP levels were at or less than 2.5 for all reefs for the entire study period (Fig. 6A). From 2002 through 2008, WP values from reefs 6 and 7 were moderate (2.0 or lower). Burreson and Ragone Calvo (1996), Ragone Calvo and Burreson (1997, 1998, 1999, 2000, 2001, 2002, 2003), Carnegie et al. (2004), and Carnegie and Burreson (2005, 2006, 2009) observed similar fall WP values at Point of Shoals in the James River from 1987 through 2008. Very low densities of oysters were observed in the Great Wicomico from 2003 through 2005 (Table 2) after removal of seed in spring 2003. Between 2003 and 2005 , a $50 \%$ decline in oyster densities was observed per year at all reefs (Table 2), even with Dermo WP of approximately 2 and essentially no MSX (Fig. 6B). This trend is consistent with the Dermo epizoology described by Andrews (1988).

The 2006 recruitment increased oyster densities throughout the river. It is notable that Dermo WP increased from 2006 through 2009 relative to levels observed between 2003 and 2005, whereas MSX was also present in the system in 2008 (Fig. 6). Annual proportional mortalities from 2006 through 2009 for 2and 3 -y-olds in upriver habitats were generally in the range of 0.7-0.9 (Table 11). Thus, higher Dermo WP in the presence of MSX resulted in higher proportional mortality of the older animals.

\section{Contribution of Mortality to the Shell Habitat Base}

Shell habitat is maintained by addition from mortality and lost to burial and taphonomic processes. Persistence of habitat is dependent on the rates of each process (Mann \& Powell 2007, Powell \& Klinck 2007, Mann et al. 2009a, Mann et al. 2009b). Mann et al. (2009b) presented high and low rate estimates for mortality contribution to the habitat depending on the timing of mortality in relation to survey collections. A parallel analysis for public oyster reefs in the Great Wicomico is presented in Table 12. Data are presented for downriver reefs, upriver reefs, and all reefs combined. Estimates of the 2006-y class contribution to shell habitat in 2007 for downriver reefs were very low $\left(<1 \mathrm{~L} / \mathrm{m}^{2} / \mathrm{y}\right.$; Table 12). Estimates of shell accretion rates (Table 12) for upriver reefs were in the range $0.7-10.1 \mathrm{~L} / \mathrm{m}^{2} / \mathrm{y}$. When both the 2006- and 2007-y classes contributed to the shell base in 2008 , the respective low and high estimates for the upriver and downriver reef combinations were 3.8-9.6 L/m $/ \mathrm{m}^{2} / \mathrm{y}$ and $0.8-3.0$ $\mathrm{L} / \mathrm{m}^{2} / \mathrm{y}$. Corresponding 2009 values were $4.3-10.1 \mathrm{~L} / \mathrm{m}^{2} / \mathrm{y}$ upriver and $0.5-1.5 \mathrm{~L} / \mathrm{m}^{2} / \mathrm{y}$ downriver. The required shell accretion rate for equilibrium with sea level rise $(3.55 \mathrm{~mm} / \mathrm{y}$ (Pyke et al. 2008)) and natural degradation processes (30\% loss $/ y$ (Powell et al. 2006, Powell \& Klinck 2007)) is $4.55 \mathrm{~L} / \mathrm{m}^{2} / \mathrm{y}$ (Mann et al. 2009a, Mann et al. 2009b) and represents a sustainable reef habitat in the absence of repletion. This value is exceeded in only the upriver reefs during 2007 through 2009 , 

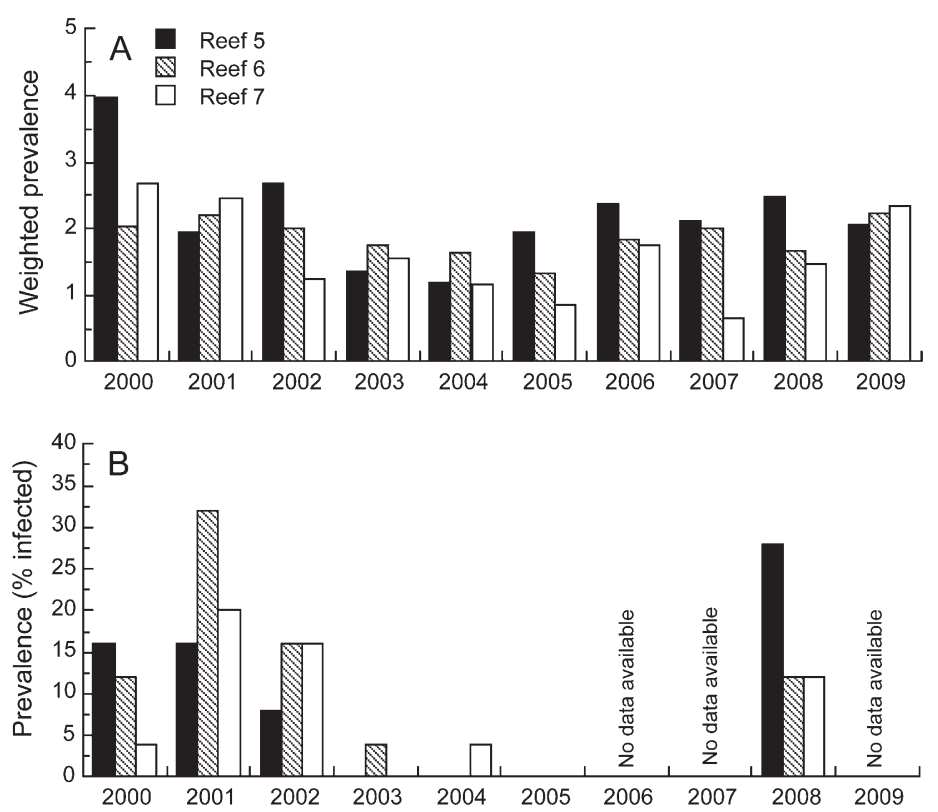

Figure 6. Weighted prevalence of Perkinsus marinus for 2000 through 2009 (A) and prevalence of Haplosporidium nelsoni for 2000 through 2005 and 2008 (B) at reefs 5,6 , and 7 . Weighted prevalence is on a scale of 0 (no disease detected) to 5 (100\% with heavy infections). Prevalence uses a scale from $0 \%$ (no oysters infected) to $100 \%$ (all oysters sampled infected).

resulting from the mortality of the 2006- and 2007-y classes. Given the current high mortality rates (Table 11), a stable habitat can only be maintained by high recruitment rates in the absence of active repletion by shell addition. The situation is exacerbated by removal of shell with seed harvests using dredges, as in 2003.

\section{Oyster Shell as Habitat on Public Reefs}

Mann et al. (2009b) describe the relationship between the shell resource and oyster populations on public oyster reefs in the James River, VA. The James River description (Mann et al. 2009 b) was for public reefs that were not subject to significant shell addition. In the James River, $92 \%$ of all data pairs were equally distributed between either the low shell + low live $(76 / 166)$ or the high shell + high live (76/166) quadrants, which were associated with live populations in, respectively, poor and good habitats. The other quadrants were poorly represented in the James River data set, and Mann et al. (2009b) suggested these alternatives as unstable conditions. The quadrant of low shell + high live would only result from a high recruitment event on limited substrate followed by high survival. The high shell + low live quadrant represents a post epizootic mortality event of adults, which is a transition toward a low shell + low live condition.

The same relationship for public reefs in the Great Wicomico River is explored in Figure 7 for the river system and in Figure 8 by reef and year. As with the James River data analyses, the median values for live shell wet weight and total shell wet weight (both measured in kilograms per square meter) divide the data into 4 quadrants corresponding to low shell + low live, low shell + high live, high shell + high live, and high shell + low live. The median value $(0.72)$ for James River live shell weight (from Mann et al. 2009b, Fig. 12B) is also presented for comparison. The Great Wicomico live shell weight median (0.34) is notably lower than that observed for the James River.

Unlike the James River reefs, the public reefs in the Great Wicomico River are subject to regular shell addition as part of replenishment activity (Table 7). The distribution of Great

TABLE 12.

Accretion rates $\left(\mathrm{L} / \mathrm{m}^{2} / \mathrm{y}\right)$ for the seven public reefs in the Great Wicomico River from 2000 through 2009.

\begin{tabular}{cccccccccccc}
\hline \hline \multicolumn{1}{c}{ Area } & Estimate & $\mathbf{2 0 0 1}$ & $\mathbf{2 0 0 2}$ & $\mathbf{2 0 0 3}$ & $\mathbf{2 0 0 4}$ & $\mathbf{2 0 0 5}$ & $\mathbf{2 0 0 6}$ & $\mathbf{2 0 0 7}$ & $\mathbf{2 0 0 8}$ & $\mathbf{2 0 0 9}$ & Mean \\
\hline Upriver & Min & 1.1 & 0.3 & $\mathbf{0 . 7}$ & 0.4 & 0.7 & 0.3 & 0.9 & 3.2 & 4.3 & 1.3 \\
& Mean & 2.1 & 0.7 & $\mathbf{2 . 8}$ & 0.8 & 1.1 & 0.2 & 3.2 & 6.4 & 7.2 & 2.7 \\
& Max & 3.2 & 1.0 & $\mathbf{4 . 8}$ & 1.1 & 1.4 & 0.1 & 5.5 & 9.6 & 10.1 & 4.1 \\
& & & & & & & & & & & \\
\multirow{2}{*}{ Downriver } & Min & 0.3 & 0.3 & $\mathbf{0 . 2}$ & 0.3 & $<0.1$ & 0.0 & $<0.1$ & 0.8 & 0.5 & 0.3 \\
& Mean & 0.5 & 0.5 & $\mathbf{0 . 6}$ & 0.5 & 0.1 & 0.0 & 0.0 & 1.9 & 1.0 & 0.6 \\
& Max & 0.8 & 0.7 & $\mathbf{1 . 0}$ & 0.6 & 0.1 & 0.0 & 0.0 & 3.0 & 1.5 & 0.9 \\
\hline
\end{tabular}

Note that seed oyster removal occurred prior to the survey in 2003, thus values for 2003 are in bold and in italics. High (Max) and low (Min) accretion rate estimates are based on time of year of mortality with respect to the survey as discussed in the text. 


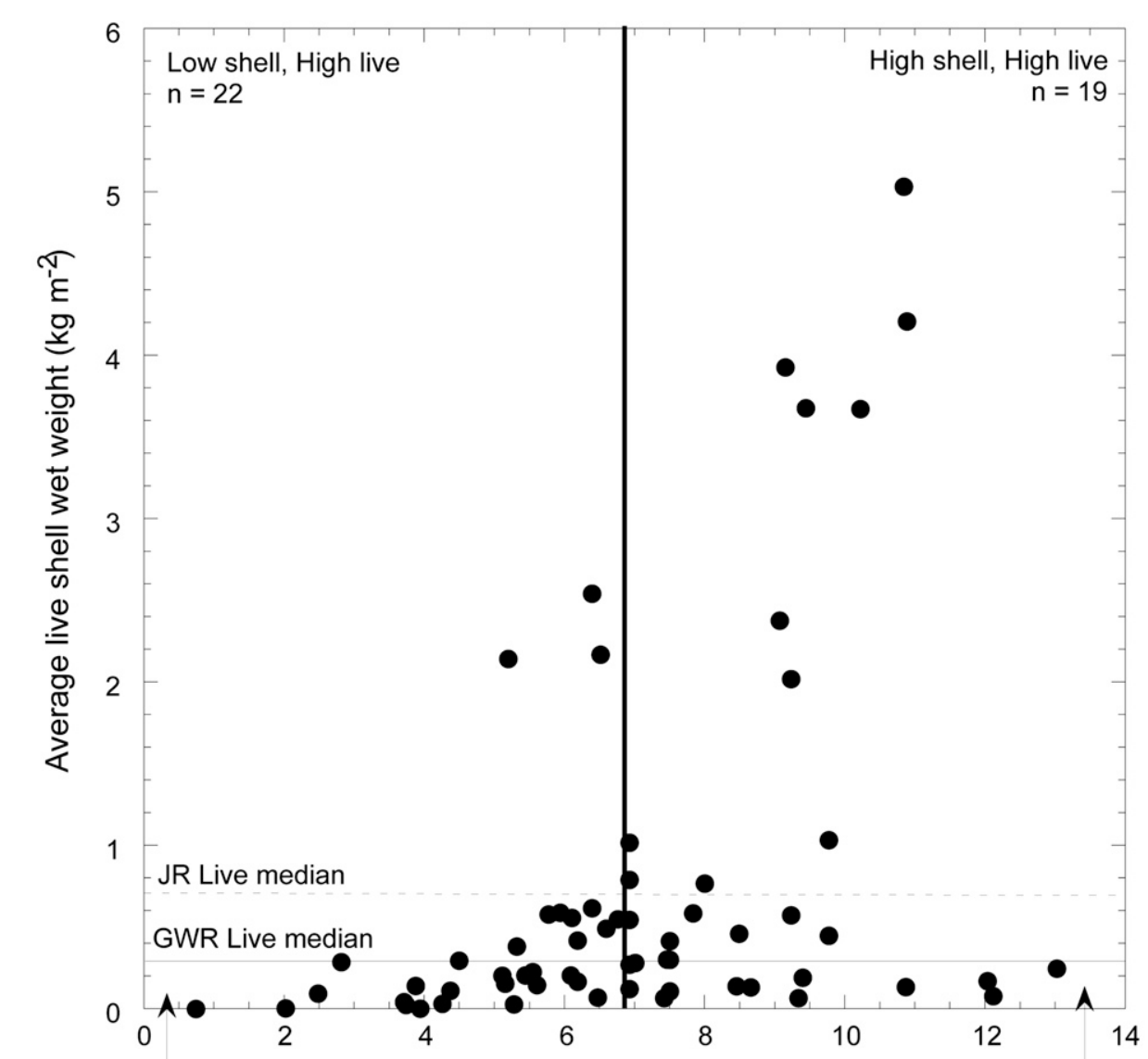
Low shell, Low live $\mathrm{n}=16$
Average total shell wet weight $\left(\mathrm{kg} \mathrm{m}^{-2}\right)$
High shell, Low live

Figure 7. The relationship of live oyster shell wet weight (median $=0.34)$ to total shell wet weight $($ median $=6.92)$ on a per-unit-area basis for all Great Wicomico River (GWR) public reefs and all years when total shell wet weight was more than $0 \mathrm{~kg} / \mathrm{m}^{2}$. Median values for the GWR are shown with solid lines. The James River (JR) live oyster shell wet weight median (0.72, Mann et al. 2009b) is included for comparison and is indicated by the dashed line. The number of data points that fall within each quadrant (live shell/total shell combination) are given.

Wicomico data among the quadrants differ markedly from that observed in the James River. Considering all Great Wicomico data together (Fig. 7), points are distributed respectively among the low shell + low live (16/70), low shell + high live (22/70), high shell + low live $(13 / 70)$, and high shell + high live $(19 / 70)$ quadrants.

The temporal sequence of the data record is illustrated in Figure 8, with the solid lines corresponding to periods of addition of shell. None of the seven reefs are stable - that is, they are consistently in the high shell + high live quadrant. Transitions to the high shell + high live condition in the absence of shell addition through replenishment occur only at reef 5 (2006 to 2007 and 2008 to 2009 following a transition to and from low shell + high live in 2008) and reef 7 in 2009, where the final live value was still modest (1.02).

Recruitment in 2006 combined with regular shell additions from 2000 through 2006 resulted in a net increase in live oysters that pushed reef 1 (Fig. 8A) from low shell + low live to high shell + high live. Reef 1 occupied the high shell + high live condition from 2006 through 2008, but declined to the low shell + high live state in 2009. The 2006 recruitment combined with the 2007 shell addition corresponded to a major transition for reefs 2,3 , and 4 from the low shell + low live to the high shell + high live state by fall 2007, where they remained through fall 2009. The position of reef 5 in the high live state from 2007 through 2009 is the direct result of the strong 2006 recruitment and subsequent maturation of surviving individuals. Despite the complicating effect of shell addition, the signals of high recruitment in 2006 (Tables 2, 5, and 8) are reflected in increasing live and total shell values in 2007. The combinations of growth and mortality maintain both live and total shell values in 2008. There are modest declines in the live shell values between 2008 and 2009 at reefs $1-4$ and maintenance at reef 5 , these changes accompany the mortality of the surviving 2006 recruits.

In contrast to the upriver reefs, reefs 6 and 7 are consistently found in the low shell + low live state (Fig. 8F and G). Shell additions (Table 7, 2009) do not appear to have any effect. The downriver reefs do not display a high recruitment signal even during years when high recruitment is observed on the upriver reefs $(2002,2006)$. The absence of this recruitment signal causes a lack of subsequent contribution to the shell resource through mortality.

The ratios between live and total shell speak to the long-term shell budget resulting from mortality and shell loss through dissolution and burial. In the exploited but otherwise minimally 

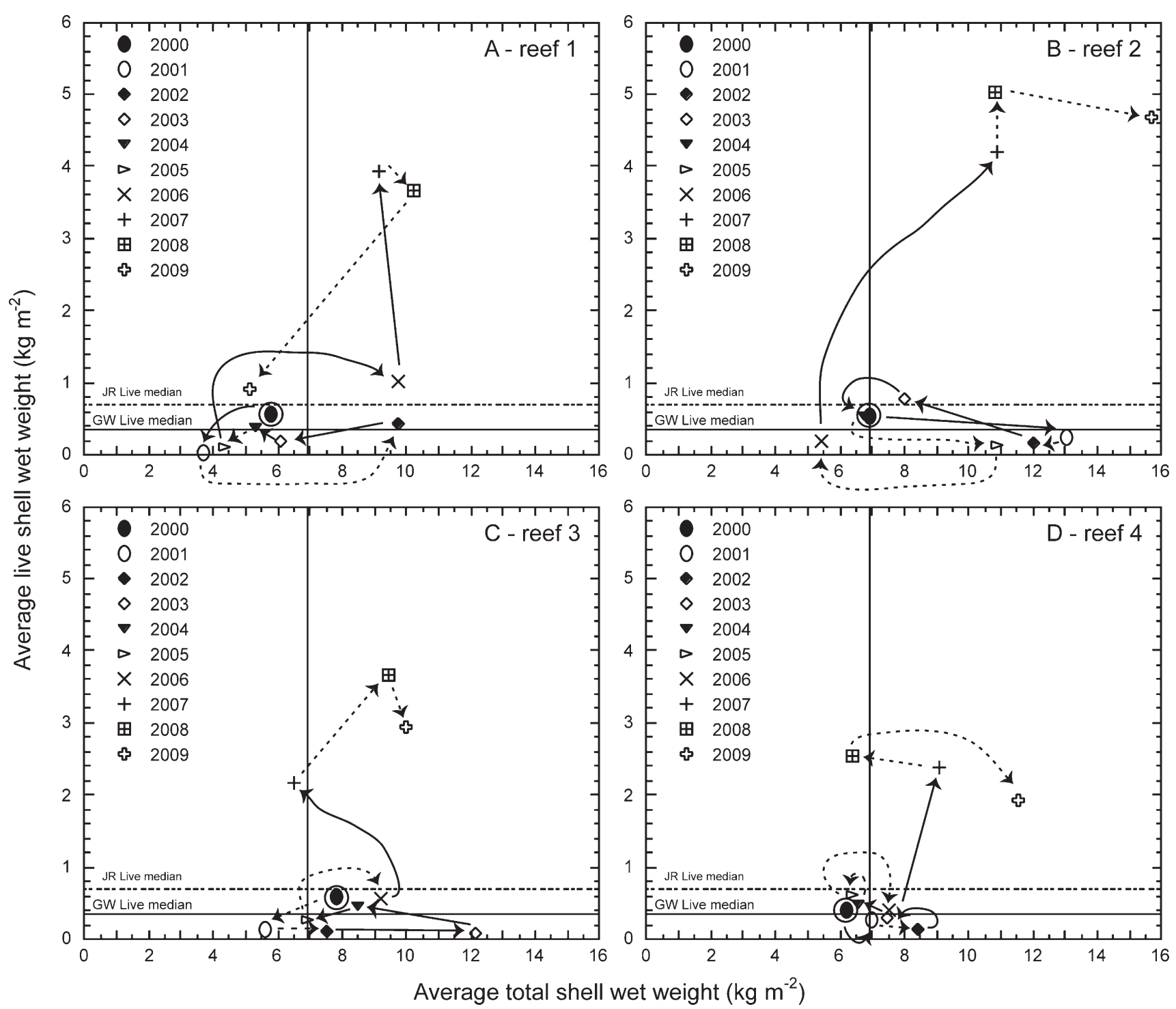

Figure 8. (A-G) Quadrant plots of average live shell wet weight versus total shell wet weight for the seven reefs from 2000 through 2009 . The derivation of the four quadrants (low shell + high live, high shell + high live, low shell + low live, and high shell + low live) follow those in Figure 7 and are based on Mann et al. (2009b). Solid arrows indicate years when shell was added to a particular reef, dashed lines represent years with no shell additions (see text for more details). GW, Great Wicomico; JR, James River.

manipulated high-density (sensu Mann et al. 2009b) James River reefs, the data in the high shell + high live quadrant present an approximate 1:3 ratio $(0.33)$ between live shell and total shell. Much lower proportions of live shell to total shell were observed in low-quality habitats, with median ratio values varying between 0.08 and 0.18 (Mann et al. 2009b, Figure 12D). Particularly low values were associated with epizootics. The intersection of the overall live shell and overall total shell median values in Figures 7 and 8 give a very low live-to-total shell ratio of 0.02 -well below that observed for low-quality James River habitats.

The limited number of points in the high shell + high live (19/ 70) quadrant in the overall plot (Fig. 7) exhibit live shell values in the $1-5 \mathrm{~kg} / \mathrm{m}^{2}$ range and total shell values in the $8-15 \mathrm{~kg} / \mathrm{m}^{2}$ range, with individual ratios of live-to-total shell in the range $0.1-0.5$. The latter ratios are commensurate with the highest values recorded on the James River reefs (Mann et al. 2009b), underscoring the value of this live-to-total shell metric to both fishery and restoration efforts.

Figure 9 presents the proportion of live shell weight to total shell volume weight by year for all reefs combined (Fig. 9A), and by reef for all years combined (Fig. 9B). The impact of the 2006 recruitment is marked in Figure 9A as the median value increases at least 5 -fold from $2005(<0.05)$ to $2006(>0.2)$. The median ratio remains more than 0.2 through 2009 . The disparity between upriver and downriver reefs is evident in Figure 9B, with the former having higher median and 75 th percentile ratio values throughout the course of the study.

The live shell-to-total shell ratio is a product of the recruitment, growth, mortality, and taphonomic loss of shell from several year classes and a useful indicator of the success of management efforts. Individual recruitment events are, 

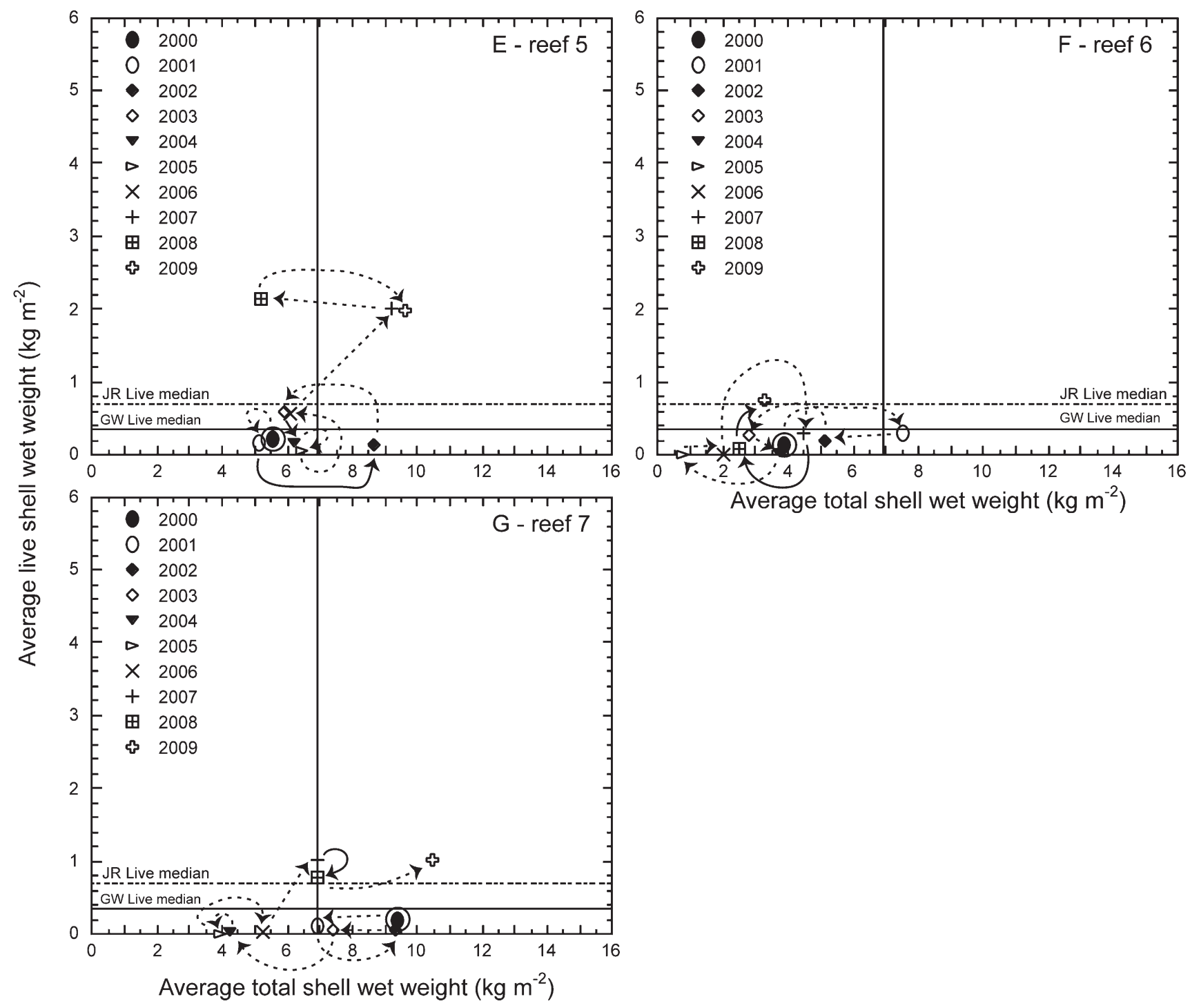

Figure 8. (Continued).

however, highly variable and unpredictable. The observed high mortality rates and resulting truncated age structure underscore the probability of long-term instability in the population, especially in the face of a succession of warm winters and dry summers that drive increased disease epizootics. These uncertainties present challenges for long-term resource management. An approach to retain a standing stock and harvest quota based on the presumption of maintenance of standing stock within well-defined proportions of carrying capacity are undermined by the instability of standing stock over timeindependent of management actions. The current data do, however, demonstrate the potential for high productivity of traditional 2-dimensional reefs augmented by modest shell planting. The efficacy of shell planting is documented by the current study. The habitat accretion rate over the $10 \mathrm{y}$ described herein approximates that to match sea level rise, but this is in large part the result of the 2006-y class. During prolonged periods when recruitment is low, a fishery management protocol that relies on shell planting to sustain habitat in concert with cost-benefit analyses based on fishery production is appropriate.

The Great Wicomico River was developed as a seed oyster production river in 1963, based on the regular replenishment of shell resource and subsequent harvest of that resource following the annual spawning and recruitment season. From 1962/1963 through $1971 / 1972$, the river produced an annual average of $1.19 \times 10^{5}$ bushels of seed oysters/y, commensurate with the annual average shell planting of $5.89 \times 10^{5}$ bushels of shell/y (Haven et al. 1978). No seed were removed in 1972/1973 and $1973 / 1974$ with only $8.31 \times 10^{3}$ bushels harvested in $1974 / 1975$ (Haven et al. 1978). The observed decline after 1972 has been attributed to the combination of Tropical Storm Agnes (June through July 1972) and low dissolved oxygen events during the summers of 1973 through 1975 (Haven et al. 1978). Seed production in the river remained low from 1974/1975 through $1983 / 1984$, with average annual production of $2.67 \times 10^{3}$ bushels of seed (Haven \& Whitcomb 1986). With the exceptions of 1998 and $2003\left(2.03 \times 10^{4}\right.$ bushels/y and $4.33 \times 10^{4}$ bushels/y, 

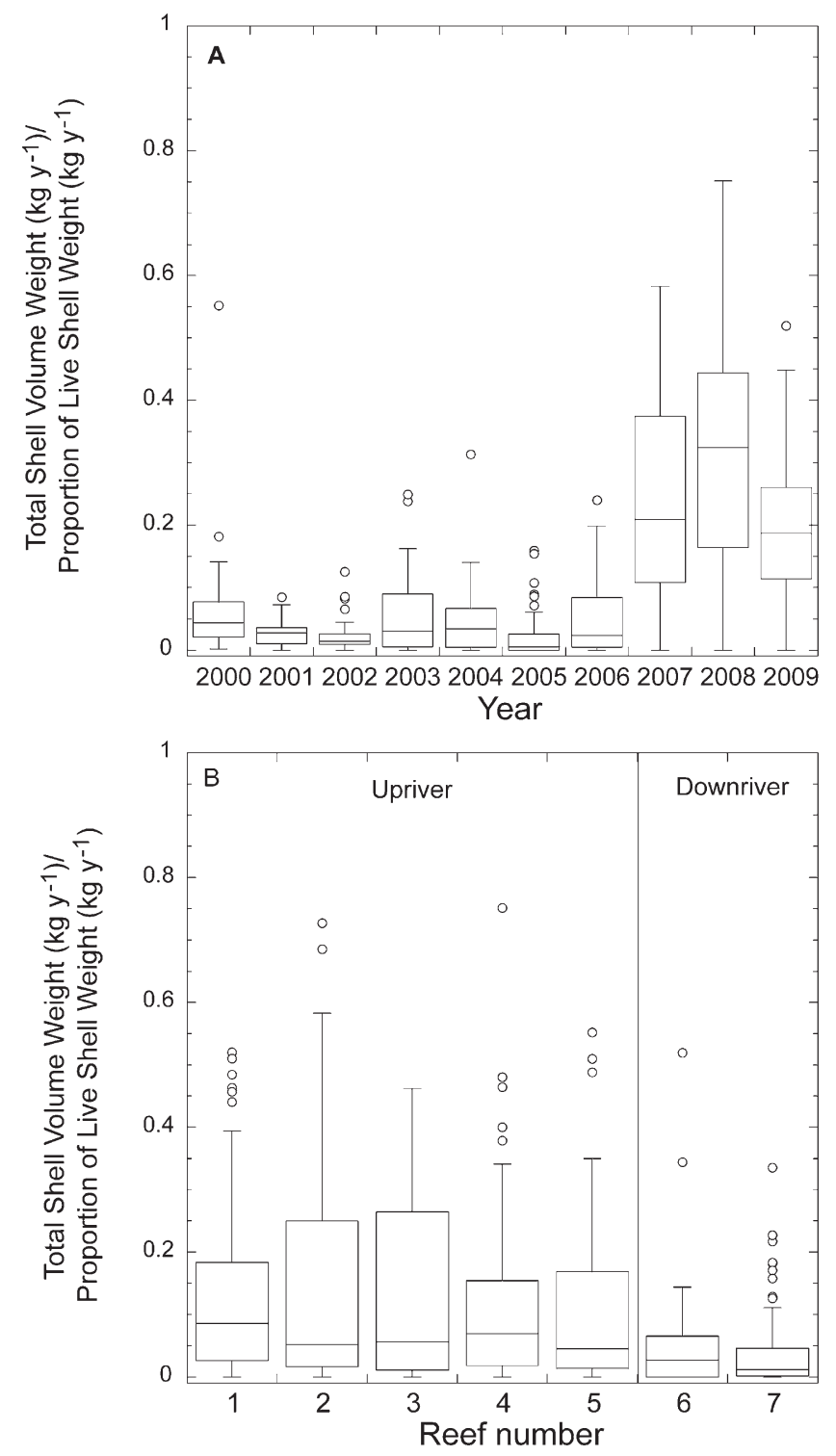

Figure 9. (A, B) The proportion of average live shell wet weight to total shell wet weight by year (A) and reef (B) as measured on the seven Great Wicomico reefs from 2000 through 2009.

respectively; unpublished VMRC annual repletion reports for these years), seed harvest in the river has remained at these levels since the mid 1980s. The potential for maintenance of selfperpetuating Great Wicomico oyster populations with regard to both live oysters and oyster shell substrate is unknown in the face of high disease mortality and the relative lack of natural cultch in the system based on low accretion rates throughout. Disease-related mortality effectively removes all individuals older than 3 y throughout the river basin. Oyster life history strategy evolved over thousands of years and is based on (1) the presumed life expectancy of 10-20 y (Powell \& Cummings 1985), (2) sequential protandry resulting in larger individuals being female, (3) a nonlinear relationship between size and fecundity (Cox \& Mann 1992), and (4) a nonlinear relationship between size and the contribution to the habitat (Mann et al. 2009a). The current situation in the Great Wicomico River, where the life expectancy is $3 \mathrm{y}$, effectively truncates the reproductive biology as well as creation of biogenic habitat.

In the absence of a way to remove the disease pressure, proactive management may offer hope for the continuation of the Great Wicomico as a productive seed river. Rotational seed harvest strategies, such as those suggested by Myers et al. (2000), which are currently under investigation in the Rappahannock River for market oysters, may provide local relief from harvest pressure if used on timescales that are relevant in terms of oyster life history and if used in synchrony with continued shell planting programs. Annual adjustment of seed quotas using values based on the previous year's fall survey density and habitat data may also be prudent.

\section{ACKNOWLEDGMENTS}

This work was supported by funds from the Commonwealth of Virginia to the Virginia Institute of Marine Science and the Virginia Marine Resources Commission, and award nos. NA06NMF4570312 and NA07NMF4570335 from the National Oceanic and Atmospheric Administration. The authors thank their colleagues, Drs. Ryan Carnegie and Eugene Burreson, for sharing unpublished data on MSX in the study location, and the many individuals who assisted in fieldwork, especially Alan Godshall, Vernon Rowe, John Ericson, Adam Crockett, and Erin Reilly. Todd Nelson designed the hydrographic monitoring station and assisted with station deployment and maintenance. This contribution is dedicated to the late Dr. Jay D. Andrews for his passionate support of preservation and restoration of the oyster resources of the Chesapeake Bay. This is contribution no. 3077 from the School of Marine Science, Virginia Institute of Marine Science.

\section{NOTES}

${ }^{1}$ The Baylor survey was commissioned in 1892 and the resulting maps by river and county became available between 1892 and 1896 . We use 1896 as the citation date because the complete map set was finished in this year.

${ }^{2}$ The bushels referred to herein are Virginia bushels (3003.9 $\mathrm{in}^{3}$ or $49.2 \mathrm{~L})$.

\section{LITERATURE CITED}

Andrews, J. D. \& J. L. Wood. 1967. Oyster mortality studies in Virginia. VI. History and distribution of Minchinia nelsoni, a pathogen of oysters in Virginia. Ches. Sci. 8:1-13.

Andrews, J. D. 1979. Pelecypoda: Ostreidae. In: A. C. Giese \& J. S. Pearse, editors. Reproduction of marine invertebrates. New York: Academic Press. pp. 293-341.

Andrews, J. D. 1988. Epizootiology of the disease caused by the oyster pathogen Perkinsus marinus and its effects on the oyster industry. Am. Fish. Soc. Spec. Publ. 18:47-63.
Andrews, J. D. 1996. History of Perkinsus marinus, a pathogen of oysters in Chesapeake Bay 1950-1984. J. Shellfish Res. 15: $13-16$.

Baylor, J. B. 1896. Method of defining and locating natural oyster beds, rocks and shoals. Oyster records (pamphlets, one for each Tidewater, Virginia, County that listed the precise boundaries of the Baylor Survey). Board of Fisheries of Virginia. 770 pp.

Bhattacharya, C. G. 1967. A simple method of resolution of a distribution into Gaussian components. Biometrics 23:115-135. 
Bros, W. E. \& B. C. Cowell. 1987. A technique for optimizing sample size (replication). J. Exp. Mar. Biol. Ecol. 114:63-71.

Burreson, E. M. \& L. M. Ragone Calvo. 1996. Epizootiology of Perkinsus marinus disease of oysters in the Chesapeake Bay with emphasis on data since 1985. J. Shellfish Res. 15:17-34.

Burreson, E. M., M. E. Robinson \& A. Villalba. 1988. A comparison of paraffin histology and hemolymph analysis of the diagnosis of Haplosporidium nelsoni (MSX) in Crassostrea virginica (Gmelin). J. Shellfish Res. 7:19-23.

Carnegie, R. \& E. M. Burreson. 2005. Status of the major oyster diseases in Virginia-2004. A summary of the annual monitoring program. Virginia Institute of Marine Science report. Gloucester Point, VA: Virginia Institute of Marine Science. $14 \mathrm{pp}$.

Carnegie, R. \& E. M. Burreson. 2006. Status of the major oyster diseases in Virginia-2005. A summary of the annual monitoring program. Virginia Institute of Marine Science report. Gloucester Point, VA: Virginia Institute of Marine Science. $14 \mathrm{pp}$.

Carnegie, R. \& E. M. Burreson. 2009. Status of the major oyster diseases in Virginia 2006-2008. A summary of the annual oyster disease monitoring program. Virginia Institute of Marine Science report. Gloucester Point, VA: Virginia Institute of Marine Science. 20 pp.

Carnegie, R., L. Ragone Calvo \& E. M. Burreson. 2004. Status of the major oyster diseases in Virginia-2003. A summary of the annual monitoring program. Virginia Institute of Marine Science report. Gloucester Point, VA: Virginia Institute of Marine Science. 14 pp.

Cox, C. \& R. Mann. 1992. Temporal and spatial changes in fecundity of Eastern oysters, Crassostrea virginica (Gmelin, 1791) in the James River, Virginia. J. Shellfish Res. 11:49-54.

Fegley, S. R. 2001. Demography and dynamics of hard clam populations. In: J. N. Kraeuter \& M. Castagna, editors. Biology of the hard clam. New York: Elsevier. pp. 383-422.

Ford, S. E. \& H. H. Haskin. 1982. History and epizootiology of Haplosporidium nelsoni (MSX), an oyster pathogen in Delaware Bay, 1957-1980. J. Invert. Pathol. 40:118-141.

Harding, J. M., R. Mann \& M. Southworth. 2008. Shell length-at-age relationships in James River oysters (Crassostrea virginica) collected four centuries apart. J. Shellfish Res. 27:1109-1115.

Hargis, W. J., Jr. 1999. The evolution of the Chesapeake oyster reef system during the Holocene Epoch. In: M. W. Luckenbach, R. Mann, \& J. A. Wesson, editors. Oyster reef habitat restoration: a synopsis of approaches. Gloucester Point, VA: Virginia Institute of Marine Science. pp. 5-24.

Hargis, W. J., Jr. \& D. S. Haven. 1999. Chesapeake oyster reefs, their importance, destruction and guidelines for restoring them. In: M. W. Luckenbach, R. Mann, \& J. A. Wesson, editors. Oyster reef habitat restoration: a synopsis and synthesis of approaches. Gloucester Point, VA: Virginia Institute of Marine Science. pp. 329-358.

Haven, D. S., W. J. Hargis, Jr. \& P. Kendall. 1978. The oyster industry of Virginia: its status, problems, and promise. Virginia Institute of Marine Science special papers in marine science no. 4. Gloucester Point, VA: Virginia Institute of Marine Science. 1024 pp.

Haven, D. S., J. P. Whitcomb \& P. Kendall. 1981. The present and potential productivity of the Baylor Grounds in Virginia. Virginia Institute of Marine Science, Special report Applied Marine Science of Ocean Engineering no. 243. Gloucester Point, VA: Virginia Institute of Marine Science. $154 \mathrm{pp}$.

Haven, D.S. and J.P. Whitcomb. 1986. The public oyster bottoms in Virginia: an overview of their size, location, and productivity. Am. Malacol. Bull. 3:17-23.

Hobbs, C. H. 2004. Geological history of the Chesapeake Bay, USA. Q. Sci. Rev. 23:641-661.

Kraeuter, J. N., S. Ford \& M. Cummings. 2007. Oyster growth analysis: a comparison of methods. J. Shellfish Res. 26:479-491.

Mann, R. \& D. A. Evans. 2004. Site selection for oyster habitat rehabilitation in the Virginia portion of the Chesapeake Bay. $J$. Shellfish Res. 23:41-49.
Mann, R., J. M. Harding \& M. Southworth. 2009a. Reconstructing precolonial oyster demographics in the Chesapeake Bay, USA. Estuar. Coast. Shelf Sci. 85:217-222.

Mann, R. \& E. N. Powell. 2007. Why oyster restoration goals in the Chesapeake Bay are not and probably cannot be achieved. $J$. Shellfish Res. 26:905-917.

Mann, R., M. Southworth, J. M. Harding \& J. Wesson. 2009b. Population studies of the native oyster, Crassostrea virginica (Gmelin), in the James River, Virginia, USA. J. Shellfish Res. 28:193-220.

Myers, R. A., S. D. Fuller \& D. G. Kehler. 2000. A fisheries management strategy robust to ignorance: rotational harvest in the presence of indirect fishing mortality. Can. J. Fish. Aquat. Sci. 57:2357-2362.

Powell, E. \& H. Cummings. 1985. Are molluscan maximum life spans determined by long-term cycles in benthic communities? Oecologica 67:177-182.

Powell, E. N. \& J. M. Klinck. 2007. Is oyster shell a sustainable estuarine resource? J. Shellfish Res. 26:181-194.

Powell, E. N., J. N. Kraeuter \& K. A. Ashton-Wilcox. 2006. How long does oyster shell last on an oyster reef? Estuar. Coast. Shelf Sci. 69:531-542.

Pyke, C. R., R. G. Najar, M. B. Adams, D. Breitburg, M. Kemp, C. Hershner, R. Howarth, M. Mulholland, M. Paolisso, D. Secor, K. Sellner, D. Wardrop \& R. Wood. 2008. Climate change and the Chesapeake Bay: state of the science review and recommendations. A report from the Chesapeake Bay Program Science and Technical Advisory Committee (STAC). Annapolis, MD. Edgewater, MD: Chesapeake Research Consortium, Inc. 59 pp.

Ragone Calvo, L. \& E. M. Burreson. 1997. Status of the major oyster diseases in Virginia 1996. A summary of the annual monitoring program. Virginia Institute of Marine Science marine resource report no. 97-2. Gloucester Point, VA: Virginia Institute of Marine Science. 25 pp.

Ragone Calvo, L. \& E. M. Burreson. 1998. Status of the major oyster diseases in Virginia 1997. A summary of the annual monitoring program. Virginia Institute of Marine Science marine resource report no. 98-1. Gloucester Point, VA: Virginia Institute of Marine Science. $24 \mathrm{pp}$.

Ragone Calvo, L. \& E. M. Burreson. 1999. Status of the major oyster diseases in Virginia 1998. A summary of the annual monitoring program. Virginia Institute of Marine Science marine resource report no. 99-3. Gloucester Point, VA: Virginia Institute of Marine Science. 15 pp.

Ragone Calvo, L. M. \& E. M. Burreson. 2000. Status of the major oyster diseases in Virginia 1999. A summary of the annual monitoring program. Virginia Institute of Marine Science marine resource report no. 00-2. Gloucester Point, VA: Virginia Institute of Marine Science. 23 pp.

Ragone Calvo, L. M. \& E. M. Burreson. 2001. Status of the major oyster diseases in Virginia 2000. A summary of the annual monitoring program. Virginia Institute of Marine Science marine resource report no. 01-8. Gloucester Point, VA: Virginia Institute of Marine Science. 21 pp.

Ragone Calvo, L. M. \& E. M. Burreson. 2002. Status of the major oyster diseases in Virginia 2001. A summary of the annual monitoring program. Virginia Institute of Marine Science marine resource report no. 02-1. Gloucester Point, VA: Virginia Institute of Marine Science. 20 pp.

Ragone Calvo, L. M. \& E. M. Burreson. 2003. Status of the major oyster diseases in Virginia 2002. A summary of the annual monitoring program. Virginia Institute of Marine Science marine resource report no. 03-2. Gloucester Point, VA: Virginia Institute of Marine Science. 35 pp.

Smith, G. F., E. B. Roach \& D. G. Bruce. 2003. The location, composition, and origin of oyster bars in Mesohaline Chesapeake Bay. Estuar. Coast. Shelf Sci. 56:391-409. 
Southworth, M., J. M. Harding \& R. Mann. 2000. Management of oyster broodstock sanctuaries in the Great Wicomico and Piankatank Rivers, Virginia: optimal use of cultch to maximize settlement. Unpublished final report from Virginia Institute of Marine Science, Gloucester Point, VA to Virginia Department of Environmental Quality, Coastal Resources Management Program. 12 pp.

Southworth, M., J. M. Harding \& R. Mann. 2001. The status of Virginia's public oyster resource 2000. Molluscan Ecology Program. Gloucester Point, VA: Virginia Institute of Marine Science. $56 \mathrm{pp}$.

Southworth, M., J. M. Harding \& R. Mann. 2002. The status of Virginia's public oyster resource 2001. Molluscan Ecology Program. Gloucester Point, VA: Virginia Institute of Marine Science. 53 pp.

Southworth, M., J. M. Harding \& R. Mann. 2003. The status of Virginia's public oyster resource 2002. Molluscan Ecology Program. Gloucester Point, VA: Virginia Institute of Marine Science. 51 pp.

Southworth, M., J. M. Harding \& R. Mann. 2004. The status of Virginia's public oyster resource 2003. Molluscan Ecology Program. Gloucester Point, VA: Virginia Institute of Marine Science. 55 pp.

Southworth, M., J. M. Harding \& R. Mann. 2005. The status of Virginia's public oyster resource 2004. Molluscan Ecology Program. Gloucester Point, VA: Virginia Institute of Marine Science. 51 pp.

Southworth, M., J. M. Harding \& R. Mann. 2006. The status of Virginia's public oyster resource 2005. Molluscan Ecology Program. Gloucester Point, VA: Virginia Institute of Marine Science. 49 pp.
Southworth, M., J. M. Harding \& R. Mann. 2007. The status of Virginia's public oyster resource 2006. Molluscan Ecology Program. Gloucester Point, VA: Virginia Institute of Marine Science. 49 pp.

Southworth, M., J. M. Harding \& R. Mann. 2008. The status of Virginia's public oyster resource 2007. Molluscan Ecology Program. Gloucester Point, VA: Virginia Institute of Marine Science. 53 pp.

Southworth, M., J. M. Harding \& R. Mann. 2009. The status of Virginia's public oyster resource 2008. Molluscan Ecology Program. Gloucester Point, VA: Virginia Institute of Marine Science. 51 pp.

Southworth, M., J. M. Harding \& R. Mann. 2010. The status of Virginia's public oyster resource 2009. Molluscan Ecology Program. Gloucester Point, VA: Virginia Institute of Marine Science. 50 pp.

Southworth, M. \& R. Mann. 1998. Oyster reef broodstock enhancement in the Great Wicomico River, Virginia. J. Shellfish Res. 17: 1101-1114.

Southworth, M. \& R. Mann. 2004. Decadal scale changes in seasonal patterns of oyster recruitment in the Virginia subestuaries of the Chesapeake Bay. J. Shellfish Res. 23:391-402.

von Bertalanffy, L. 1938. A quantitative theory of organic growth (inquiries on growth laws. II). Hum. Biol. 10:181-213.

Woods, H., W. J. Hargis, Jr., C. H. Hershner \& P. Mason. 2005. Disappearance of the natural emergent 3-dimensional oyster reef system of the James River, Virginia, 1871-1948. J. Shellfish Res. 24:139-142. 